\title{
THE SUBTRIBE THLADIANTHINAE (CUCURBITACEAE) IN INDOCHINA AND MALESIA
}

\author{
W.J.J.O. DE WILDE \& B.E.E. DUYFJES \\ Nationaal Herbarium Nederland, Universiteit Leiden branch, P.O. Box 9514, \\ 2300 RA Leiden, The Netherlands; e-mail: dewilde@nhn.leidenuniv.nl
}

\begin{abstract}
SUMMARY
Of the seven genera which constitute the subtribe Thladianthinae five occur in our area. Full descriptions are given of the genera and species not treated previously by us. Two new species have been described: Sinobaijiania smitinandii W.J. de Wilde \& Duyfjes and Thladiantha angustisepala W.J. de Wilde \& Duyfjes.
\end{abstract}

Key words: Thladianthinae, Cucurbitaceae, SE Asia, taxonomy.

\section{INTRODUCTION}

Since the reassessment by Jeffrey \& De Wilde (2006) of the 7 genera in the subtribe Thladianthinae Pax (1889), it appears that 5 genera of the subtribe occur in the area under consideration: Baijiania, Momordica, Sinobaijiania, Siraita and Thladiantha. The other two genera are Indofevillea (India, Bhutan, China) and Microlagenaria (Africa). The subtribe is characterized by the presence of basal ventral scales on the petals. It can be divided into a group of genera having the tendrils unbranched or branched and then spiralling both above and below the point of branching and a group of genera having the tendrils unbranched or branched and then spiralling only above the point of branching. In the present account two recently treated genera, Baijiania (a monotypic genus, endemic to Borneo; see De Wilde \& Duyfjes, 2003) and Momordica (widespread, c. 50 species, of which 6 in Malesia and 3 in Thailand; see De Wilde \& Duyfjes, 2002) are left out; the remaining ones are described here. A classification of the genera with an enumeration and short discussion of the species in each genus can be found in Jeffrey \& De Wilde (2006).

\section{KEY TO THE GENERA}

1a. Tendrils distally 2-branched, spiralling both above and below the point of branching (tendrils rarely simple but then spiralling almost throughout their length)... . 2

b. Tendrils medianly or proximally 2-branched, spiralling only above the point of branching, or tendrils unbranched and then spiralling only in the upper part or sometimes spiralling almost throughout their length (T. cordifolia, p.p.).... . 4

2a. Basal adaxial petal scales (at least in male flowers) absent. Receptacle-tube deep. Stamens inserted towards the apex in the tube. - Borneo ......... Baijiania 
b. Basal adaxial petal scales present. Receptacle-tube shallow. Stamens inserted towards the base of the tube ........................ 3

3a. Hairs of leaves and stem blackish glandular. Anther thecae sigmoid. Disc absent. Seeds with (broad) wing-like double or triplicate margin. - Widespread in SE Asia, also in West Malesia. . . . . . . . . . . . . . . . 2. Siraitia

b Hairs of leaves and stem not glandular. Anther thecae straight or \pm curved, not sigmoid. Disc in receptacle-tube merely the thickened basal part. Seeds unwinged.

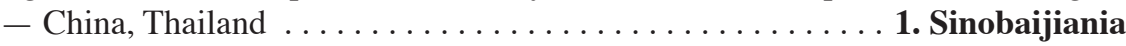

4a. Stamens 2 or 3. Anther thecae strongly folded or sigmoid. Disc absent or inconspicuous. - Widespread in Africa and Asia ...............Momordica

b. Stamens 5 (rarely 3). Anther thecae straight or curved. Disc obvious, half-globose and \pm asymmetrically inserted. - E \& SE Asia, 1 species extending into West Malesia

3. Thladiantha

\section{SINOBAIJIANIA}

Sinobaijiania C. Jeffrey \& W.J. de Wilde (2006) 769. - Type: Sinobaijiania yunnanensis (A.M. Lu \& Zhi Y. Zhang) C. Jeffrey \& W.J. de Wilde.

Siraitia Merr. subg. Microlagenaria auct. non (C. Jeffrey) A.M. Lu \& Zhi Y. Zhang: A. M. Lu \& Zhi Y. Zhang (1984) 30, p.p., excluding the type.

Siraitia auct. non Merr.: H.Y. Liu (1993) 865, p.p., excluding the type.

Baijiania auct. non A.M. Lu \& J.Q. Li: A.M. Lu \& J.Q. Li in Li (1993) 45-55, p.p., excluding the type.

Herbaceous climbers with small or large spherical (totally or partly subterraneous) tuber; dioecious; plant more or less stiff-hairy to glabrescent, without glandular hairs. Probract absent on sterile nodes, not obvious in inflorescences. Tendrils distally 2-branched, spiralling both above and below point of branching or simple. Leaf-blades ovate to narrowly elliptic, simple, not lobed to shallowly 3-5-lobed, green on drying. Flowers yellow. Male flowers few in simple or branched racemes; pedicels articulate towards apex; receptacle-tube very shallow, saucer- or cup-shaped, without obvious basal disc, although wall of receptacle-tube thickened basally; sepals ovate to narrowly triangular, acute; petals longer than sepals, (narrowly) ovate to (narrowly) elliptic, each or some with an incurved basal adaxial scale; stamens 5, inserted near rim of receptacle-tube, all solitary or two in pairs and one solitary, filaments long, thecae straight or curved. Female flowers solitary or up to 3, pedunculate, similar to male flowers; stigmas reniform; staminodes 5. Fruits fleshy, indehiscent, narrowly ellipsoid to globose, 2.5-8.5 cm long, smooth; pulp not sweet. Seeds numerous, ovoid, apex obtuse or truncate, smooth, not ornamented, not margined.

Taxonomy - Sinobaijiania was described after it appeared that the type of the genus Baijiania A.M. Lu \& J.Q. Li in Li (1993), Baijiania borneensis (Merr.) A.M. Lu \& J.Q. Li, represents a monotypic genus confined to Borneo (De Wilde \& Duyfjes, 2003). The remaining species which were placed in Baijiania by A.M. Lu \& J.Q. Li in Li (1993) have been combined in Sinobaijiania by Jeffrey \& De Wilde (2006). The genus Sinobaijiania now comprises one species in Thailand, S. smitinandii, here newly described, and three species in China, of which S. yunnanensis also occurs in Laos:

1. Sinobaijiania yunnanensis (A.M. Lu \& Zhi Y. Zhang) C. Jeffrey \& W.J. de Wilde, 2006. - Siraitia borneensis (Merr.) A.M. Lu \& Zhi Y. Zhang var. yunnanensis A.M. 
Lu \& Zhi Y. Zhang (1984) 31. - Baijiania yunnanensis (A.M. Lu \& Zhi Y. Zhang) A.M. Lu \& J.Q. Li in Li (1993) 51. - Type: S. G. Wu 307 (KUN), China, Yunnan. Thladiantha sessilifolia Hand.-Maz. var. longipes A.M. Lu \& Zhi Y. Zhang (1981) 77. - Type: Y.H. Li 11954 (PE, not seen).

Sinobaijiania yunnanensis is here considered to occur in northern Laos on the evidence of a sterile specimen: Poilane 2 (P). On the label the very large, half-subterranean tuber, typical for this species, is discussed.

2. Sinobaijiania decipiens C. Jeffrey \& W.J. de Wilde (2006) 769. - Type: S.K. Lau 27136 (PE, not seen), China, Yunnan.

3. Sinobaijiania taiwaniana (Hayata) C. Jeffrey \& W.J. de Wilde (2006) 770. - Thladiantha taiwaniana Hayata (1911) 119. - Siraitia taiwaniana (Hayata) A.M. Lu \& Zhi Y. Zhang (1984) 31. - Baijiania taiwaniana (Hayata) A.M. Lu \& J.Q. Li in Li (1993) 52. - Type: Nakahara 340 (TI, not seen), Taiwan.

1. Sinobaijiania smitinandii W.J. de Wilde \& Duyfjes, spec. nov. - Fig. 1-3; Plate $2 b-d$

A congeneribus omnibus foliis breviter petiolatis, fructibus fusiformibus $6-8.5 \mathrm{~cm}$ longis distinguenda. - Typus: Phonsena, De Wilde \& Duyfjes 3910 (holo BKF; iso L), Thailand (male flowers).

Climber, 2-10 m long, with one annual shoot from a subterraneous perennial tuber c. $10 \mathrm{~cm}$ diam.; stem 5-angular, 2-3 mm thick. Tendrils finely hairy. Leaves: blade narrowly ovate, $7-20$ by $4-8 \mathrm{~cm}$, base narrowly cordate, apex acute, margin entire with sparse minute teeth, short hairy, cystoliths not obvious; petiole $1-3(-4) \mathrm{cm}$ long, both surfaces soft hairy. Inflorescences in male a peduncled bracteate raceme, $1-5 \mathrm{~cm}$ long, peduncle $0.5-1 \mathrm{~cm}$ long, with 5-15 closely set flowers; bracts imbricate, narrowly wedge-shaped, $5-10$ by $2-5 \mathrm{~mm}$, at apex incised; female flowers solitary on the nodes. Male flowers: wholly finely soft hairy, hairs $0.1 \mathrm{~mm}$ long; pedicel slender, c. $5 \mathrm{~mm}$ long; receptacle-tube saucer-shaped, c. 2.5 by $5 \mathrm{~mm}$, the basal half thickened, slightly 3-lobed in the centre, c. 1 by $2 \mathrm{~mm}$, and towards the margin lowly 10-ribbed; sepals valvate, ovate to narrowly elliptic, c. 6 by $2.5 \mathrm{~mm}$, apex acute-acuminate; petals imbricate in bud, narrowly elliptic, c. 10 by $5 \mathrm{~mm}$, apex broadly obtuse, inside with minute papillose gland-hairs, mouth of the receptacle-tube closed by 4 (or 5) carnose scales of unequal size, 1-2 mm long; stamens: two in pairs and one solitary, filaments c. $3 \mathrm{~mm}$ long, erect but at apex somewhat curved, minutely hairy, anthers dorsifixed, \pm extrorse, ellipsoid, c. 1.5 by $1 \mathrm{~mm}$, not or hardly curved. Female flowers: pedicel 10-20 mm long; ovary narrowly ellipsoid, c. 12 by $4 \mathrm{~mm}$, densely patently hairy, hairs 1-2 mm long; sepals somewhat carnose, c. 6 by $3-4 \mathrm{~mm}$; petals ovate to narrowly elliptic, $6-7$ by c. $3.5 \mathrm{~mm}$, acute, inside densely minutely gland-hairy; petal scales 5 , minute (less than $0.5 \mathrm{~mm}$ long); style column c. $3 \mathrm{~mm}$ long, style-arms c. $0.5 \mathrm{~mm}$ long, stigmas broadly horseshoe-shaped, almost smooth, c. $1.5 \mathrm{~mm}$ diam.; staminodes prominent, erect, all solitary, c. $1.5 \mathrm{~mm}$ long, hairy, at apex with anther-like appendage (always?). Fruits broadly-fusiform, $6-8.5$ by $2.5-3.5 \mathrm{~cm}$, with sparse hairs $1-2 \mathrm{~mm}$ long; fruit pulp creamy-white; fruiting pedicel 1-2 cm long. Seeds (broadly) ovoid, c. 6 by $4-5$ by $2.5-4 \mathrm{~mm}$, little or moderately compressed, pale brown.

Field-notes - Rare plant. Tuber ellipsoid or depressed globose, brown. Male bracts persistent. 
Distribution - Endemic to Thailand (NE, SW, C, SE).

Habitat \& Ecology - Edges of mixed deciduous forest, evergreen forest, and open forest; often near streams, on good soil; 100-370 m altitude. Flowering: May to October; fruiting: June to August.

Vernacular - Buap khon.

Etymology - Named after Tem Smitinand (1920-1995), Thai forest botanist, who made one of the first collections of this species.

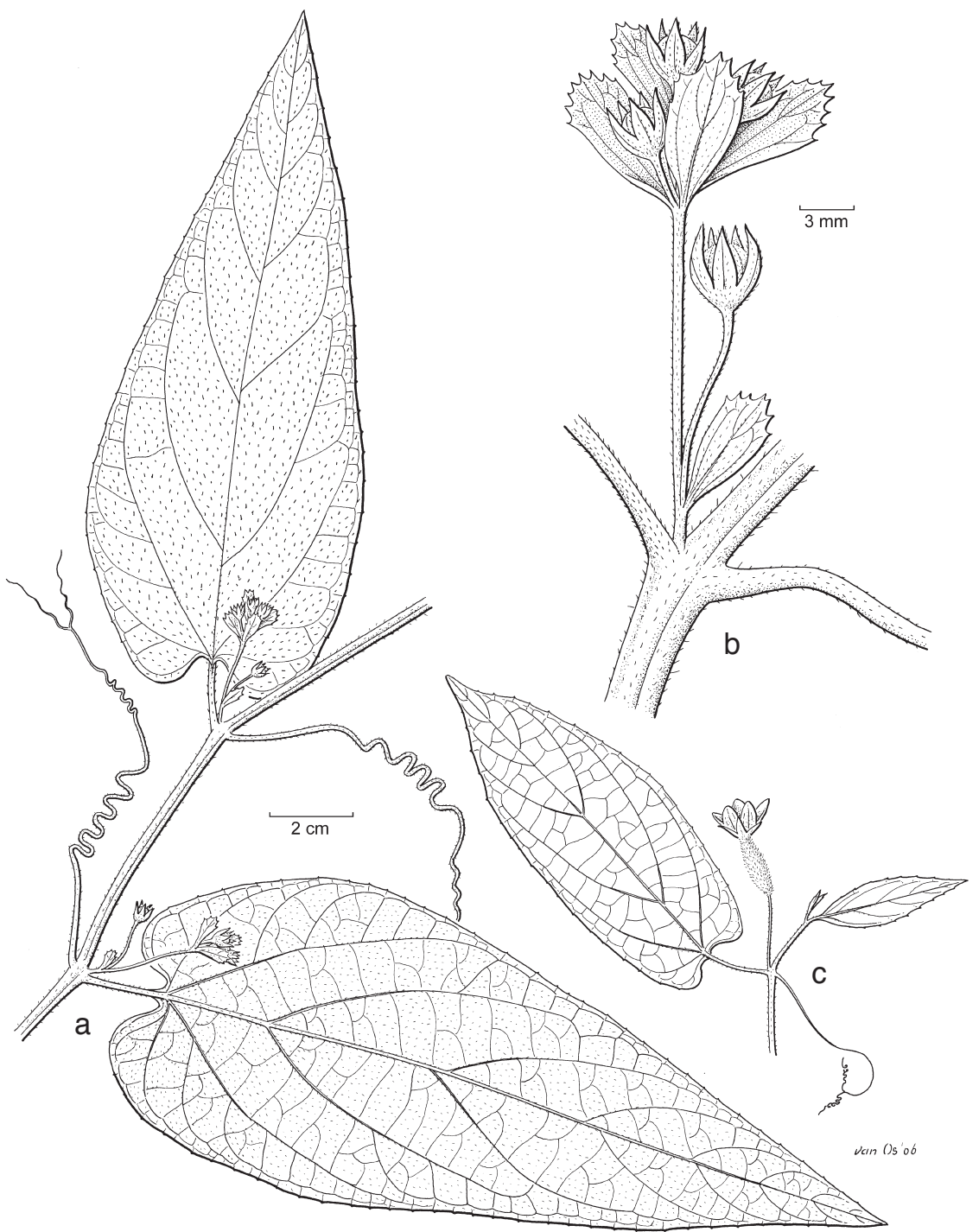

Fig. 1. Sinobaijiania smitinandii W.J. de Wilde \& Duyfjes. a. Portion of male flowering twig; b. node with male inflorescence; c. apex of shoot with one solitary female flower (a, b: Phonsena et al. 3820; c. Phonsena et al. 4588). 
Notes - 1. Several collections of Sinobaijiania from the Kunming Herbarium were sequenced by Hanno Schäfer (Munich). He found (pers. comm.) that the isotype of Siraitia borneensis (Merr.) A.M. Lu \& Zhi Y. Zhang var. lobophylla A.M. Lu \& Zhi Y. Zhang, which was placed in the synonymy of Sinobaijiania yunnanensis by Jeffrey \& De Wilde (2006), comes very close to Sinobaijiania smitinandii and most likely is not Sinobaijiania yunnanensis. The findings need further study.

2. The collection Put 18 (E, K), from Kanburi, is deviating in its more slender male inflorescences.
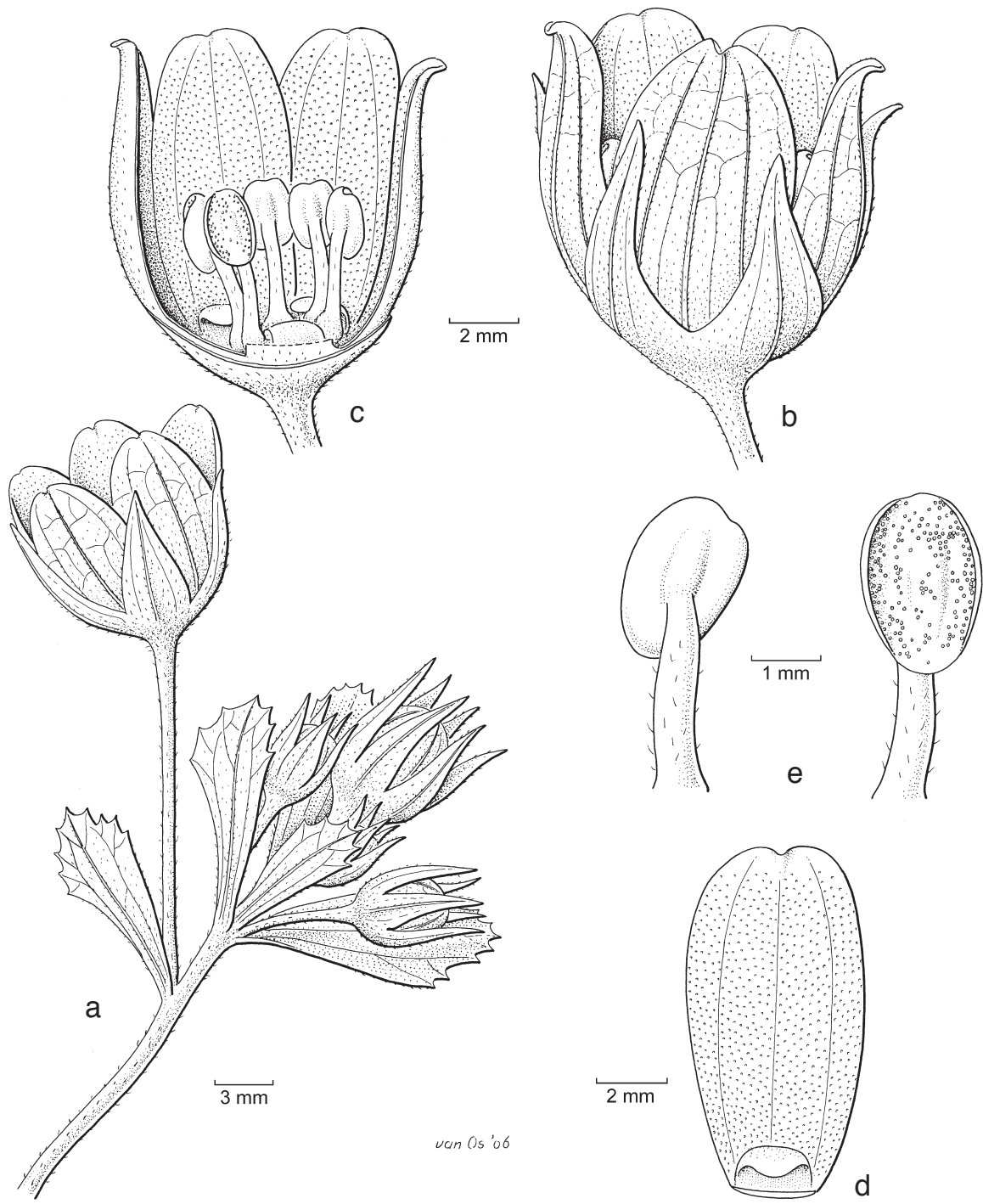

Fig. 2. Sinobaijiania smitinandii W.J. de Wilde \& Duyfjes. a. Apex of male inflorescence; b, c. male flower, from outside and opened, respectively; d. male petal with basal scale; e stamens (all: $D e$ Wilde \& Duyfjes 22298). 
Specimens examined:

De Wilde et al. 22127, 22132, 22298 (male); Koonthunthod 538 (male); Panyarat s.n. (fruit), 131; Phonsena 3747, 3802 (male), 3819 (male), 3820 (male), 4588 (female), 4610 (fruit); Phonsena et al. 3903, 3910, 4629 (male); Pooma et al. 2689; Put 18; Smitinand 8136 (type).
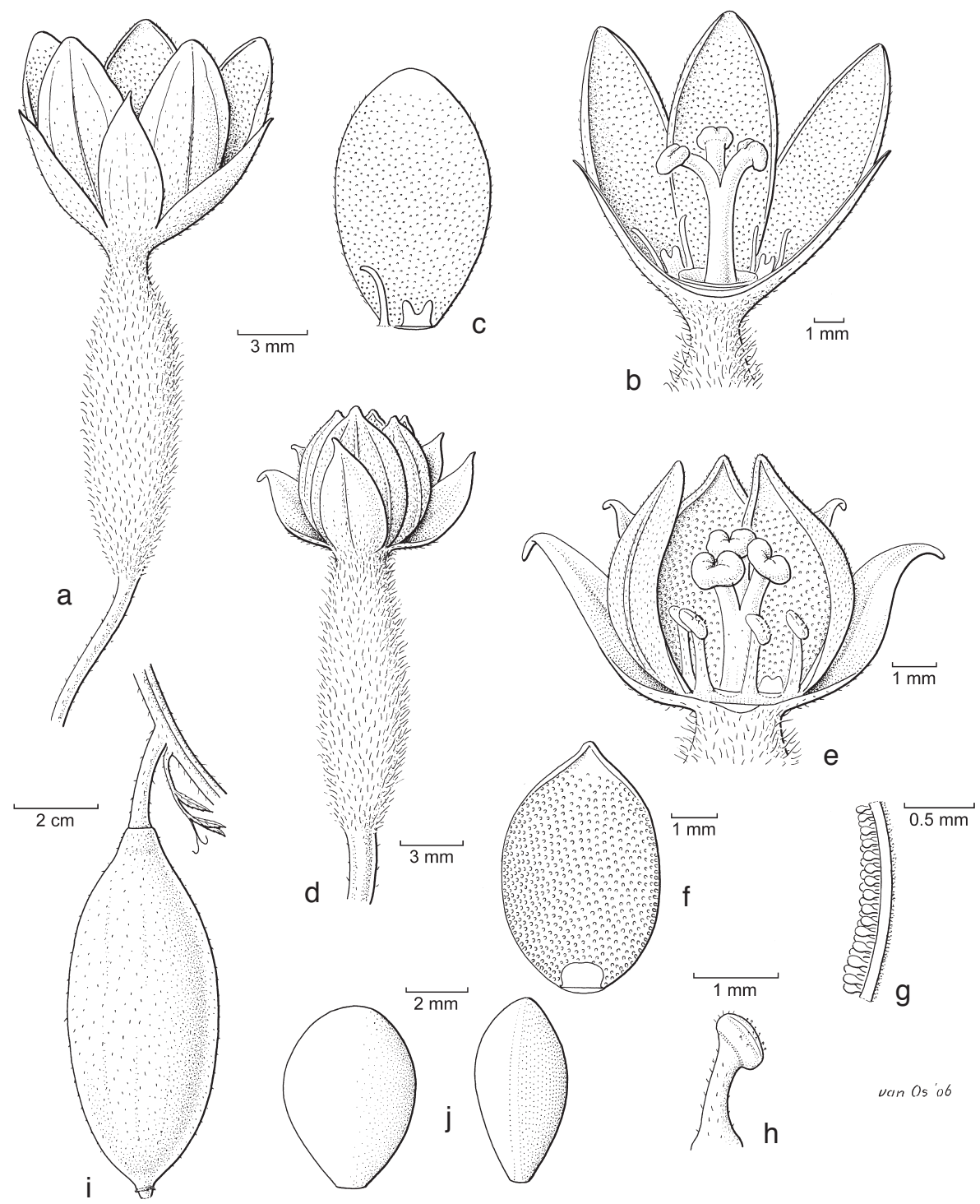

Fig. 3. Sinobaijiania smitinandii W.J. de Wilde \& Duyfjes. a, b. Female flower, from outside and opened, respectively; c. female petal with basal scale and staminode; d, e. female flower, from outside and opened, showing style and staminodes, respectively; f. female petal with basal scale; g. detail of adaxial surface of petal with papillose gland-like hairs; h. staminode; i. fruit; j. seeds (a-c: Phonsena et al. 4588; d-h: Panyarat s.n. (21-07-2004); i, j: Phonsena s.n. (17-06-2003)). 


\section{SIRAITIA}

Siraitia Merr. (1934) 200; C. Jeffrey (1980a) 25; (1982) 737; A.M. Lu \& Zhi Y. Zhang (1984) 29, p.p., for subgenus Siraitia; A.M. Lu \& J.Q. Li in Li (1993) 45-55; S.K. Chen (1995a) 310-314. - Type: Siraitia silomaradjae Merr.

Neoluffa Chakrav. (1952) 895; (1959) 70. - Type: Neoluffa sikkimensis Chakrav. [type: King s.n., 12 May 1876 (CAL, not seen), Sikkim].

Thladiantha Bunge subg. Microlagenaria C. Jeffrey (1962) 363, p.p., excluding the type (which is the genus Microlagenaria (C. Jeffrey) A.M. Lu \& J.Q. Li, from Africa).

Herbaceous climbers with sub- or supra-terraneous tuber (always?); dioecious; leaves and stems with obvious blackish glandular hairs. Probract absent or not obvious. Tendrils distally 2-branched, spiralling both below and above point of branching, although basal portion straight. Leaf-blades ovate, simple, not lobed, green on drying. Flowers yellow. Male flowers in racemes or panicles; receptacle-tube rather shallow, conspicuously coarsely anastomosing-veined externally; without obvious disc, although receptacle-tube with thickened bottom; pedicels articulate towards apex; sepals \pm triangular; petals longer than sepals, (narrowly) elliptic or (narrowly) ovate, two or all with an incurved basal adaxial scale; stamens 5, inserted in or near the throat of receptacletube, in two pairs and one solitary, filaments long, thecae sigmoid, the connective frequently with conspicuous papillae. Female flowers solitary or rarely few in a short raceme; style-arms largely free, stigmas \pm 2 -lobed or reniform; staminodes 5, glandular. Fruits \pm fleshy, indehiscent, subglobose to cylindric, rather large (5-11 cm long), smooth; pulp sweet (always?). Seeds numerous, broadly ovate or subcircular in outline, compressed, with broad finely radiatingly ribbed, corky, double or 3-ridged margin.

A genus of three or four species distributed in NE India, S \& C China, Indochina and West Malesia (Sumatra, Peninsular Malaysia, Java, Borneo); 1 species in Thailand, 1 species in Malesia.

\section{KEY TO THE SPECIES OF SIRAITIA IN THAILAND AND MALAYSIA}

1a. Calyx lobes \pm narrowly triangular, with (long-)acute-acuminate apex. Seed-margin 2-ridged. Fruit-pulp sweet. - Fruits introduced from China . . 1. S. grosvenorii

b. Calyx lobes broadly triangular with (sub)acute apex. Seed-margin 3-ridged. Fruitpulp not sweet (?). - Plant growing wild .......... 2. S. siamensis

1. Siraitia grosvenorii (Swingle) A.M. Lu \& Zhi Y. Zhang - Fig. 4c-e

Siraitia grosvenorii (Swingle) A.M. Lu \& Zhi Y. Zhang (1984) 29. - Momordica grosvenorii Swingle (1941) 198, plate 1, 2, as 'grosvenori'. - Thladiantha grosvenorii (Swingle) C. Jeffrey (1979) 393. - Type: Taam Ying-wah 1 (iso A), China, Kwangsi.

Note - Although Siraitia grosvenorii does not occur in our region it is mentioned here because its sweet, medicinally used fruits can be found in Chinese shops and local markets in Thailand. 


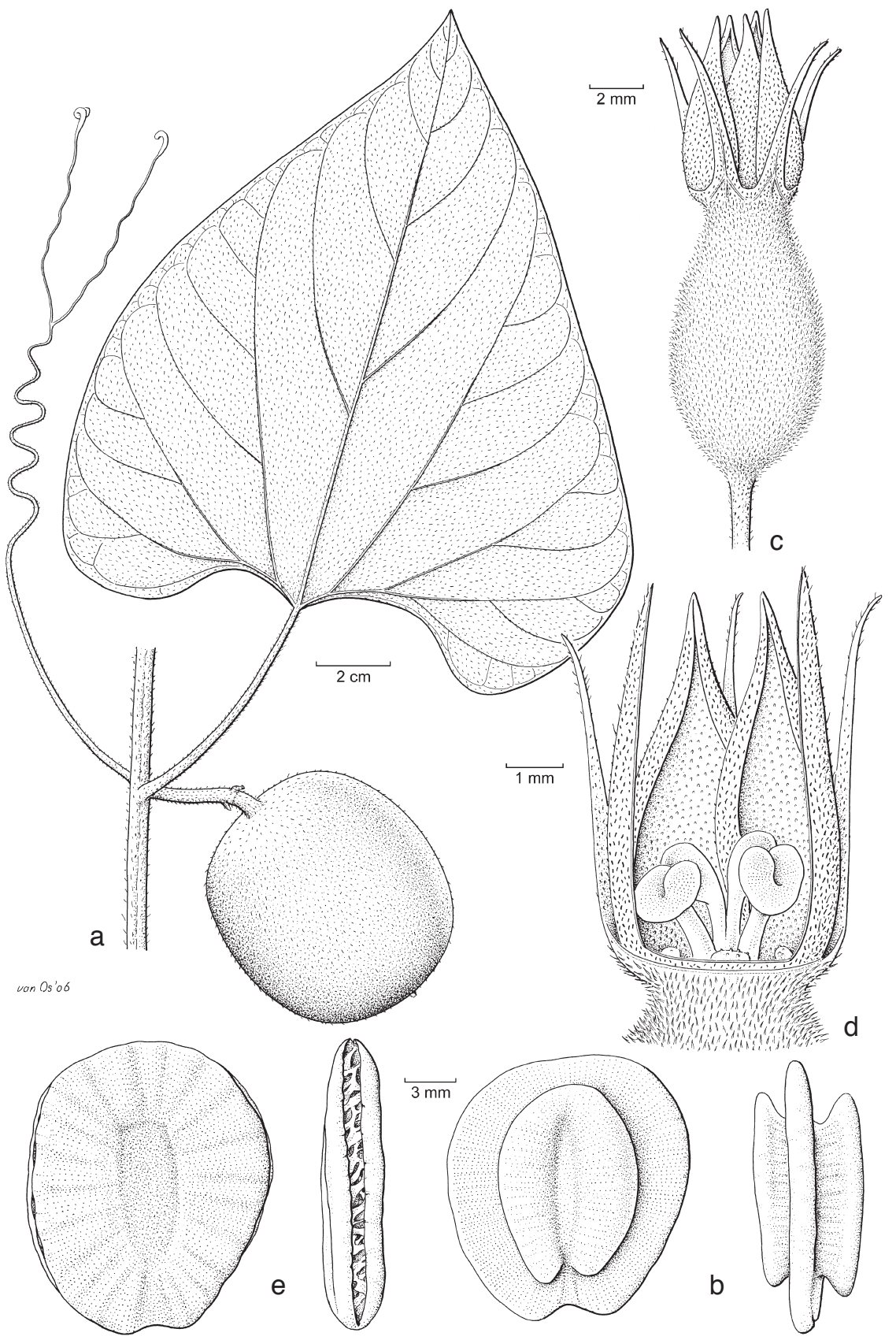

Fig. 4. a, b: Siraitia siamensis (Craib) S.Q. Zhong \& D. Fang var. siamensis. a. Portion of branch with fruit; b. seed, seen from above and laterally. - c-e: Siraitia grosvenorii (Swingle) A.M. Lu \& Zhi Y. Zhang. c. Immature female flower; d. ditto, opened; e. seed, seen from above and laterally (a: K. Larsen, S. Larsen, Nielsen \& Santisuk 32487; b: Van Beusekom \& Phenghklai 918; c, d: Zhang Guicai 445; e: De Wilde, Duyfjes \& Pruesapan 22306). 


\section{Siraitia siamensis (Craib) S.Q. Zhong \& D. Fang}

Siraitia siamensis (Craib) S.Q. Zhong \& D. Fang (1984) 23; C. Jeffrey (1980a) 26, comb. nov. provis.; A.M. Lu \& Zhi Y. Zhang (1986a) 164, f. 43: 8-12; S.K. Chen (1995a) 311, f. 81: 1-9. - Thladiantha siamensis Craib (1914) 7; (1931) 759; Cogn. (1916) 51; Keraudren (1975) 34, f. 10: 5-7. - Type: Kerr 1171 (holo K; iso E), Thailand, Doi Suthep.

Momordica tonkinensis Gagnep. (1918b) 376; (1921) 1072. - Type: Eberhardt 4273 (holo P; iso P), North Vietnam.

Climber with one (or few) shoots to 5(-10) $\mathrm{m}$ long from a supra-terranean club-shaped or ovoid tuber to $25 \mathrm{~cm}$ diam.; leafy stem subterete or \pm angular, 3(-6) mm thick, with long or short hairs. Tendrils finely pubescent. Leaves: blade ovate, $9-30$ by $8-22 \mathrm{~cm}$, base deeply cordate, apex acute-acuminate, margin sparsely minutely dentate, upper surface sparsely appressed-hairy (cystoliths not obvious), lower surface variously pubescent and with many black glandular hairs; petiole 4-10 cm long, long-hairy. Male inflorescences a peduncled raceme or loose panicle 2 (or 3) times branched, (10-)20-25 $\mathrm{cm}$ long, the flowers \pm crowded at the apex of inflorescence branches; bracts minute, 1(-2) mm long or mostly absent; peduncle 5-13 cm long; all parts finely pubescent; female flowers solitary (or 2) in a reduced raceme to $1 \mathrm{~cm}$ long. Male flowers: finely pubescent; pedicel 15-30 mm long; receptacle-tube bowl-shaped, shallow, c. 10 by $5 \mathrm{~mm}$; disc not obvious, but bottom of receptacle-tube \pm thickened; sepals triangular, c. 5 by $5 \mathrm{~mm}$, apex blunt, acute or acute-acuminate, receptacle and sepals outside with conspicuous anastomosing-netted veins; petals elliptic to narrowly elliptic or longtriangular, (10-)15 mm long, 4-8 $\mathrm{mm}$ wide at base, apex obtuse, obtuse or (long-) acute, inner surface finely papillose, at base with 2 large and 2 or 3 smaller scales, these together with the thickened bases of the filaments concealing the hollow of the receptacle-tube; stamens erect or \pm curved, filaments 5-7 mm long, anthers sigmoid one arm shorter, c. $4 \mathrm{~mm}$ long, the connective slightly enlarged and set with minute swollen appendages (oil glands?). Female flowers: pedicel 10(-20) mm long; ovary ovoid-ellipsoid, c. $10 \mathrm{~mm}$ long, hairy; perianth as in male flowers; style (China) 4-5 mm long, 3-lobed. Fruit solitary, subglobose, 5-8 cm diam., hairy, partly glabrescent; pericarp thin, c. $1 \mathrm{~mm}$ thick, woody; fruit pulp whitish, enclosing numerous densely packed seeds. Seeds c. $15 \mathrm{~mm}$ diam., pale brown, corky, with broad triplicate rim, the middle rim largest, seed without rim narrowly ellipsoid, c. $8 \mathrm{~mm}$ long.

Field-notes - Young leaves and twig apices purplish reddish.

Distribution - Widespread, from S China through Indochina (Thailand, type) into West Malesia (Peninsular Malaysia, Sumatra, Borneo, Java).

\section{KEY TO THE VARIETIES}

1a. Male inflorescences 2 (or 3) times branched, c. $20 \mathrm{~cm}$ long. Sepals of male flowers acute-acuminate, rather open in bud. Petals narrowly triangular, c. $4 \mathrm{~mm}$ wide at

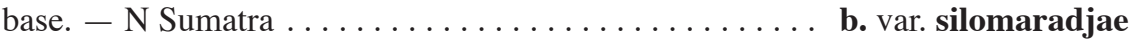

b. Male inflorescences simple or once branched, 10-20 cm long. Sepals of male flowers obtuse or sub-acute, connivent and closing the bud before anthesis. Petals elliptic, 4-8 $\mathrm{mm}$ wide at base with rounded or blunt apex. - Widespread . . . . . 

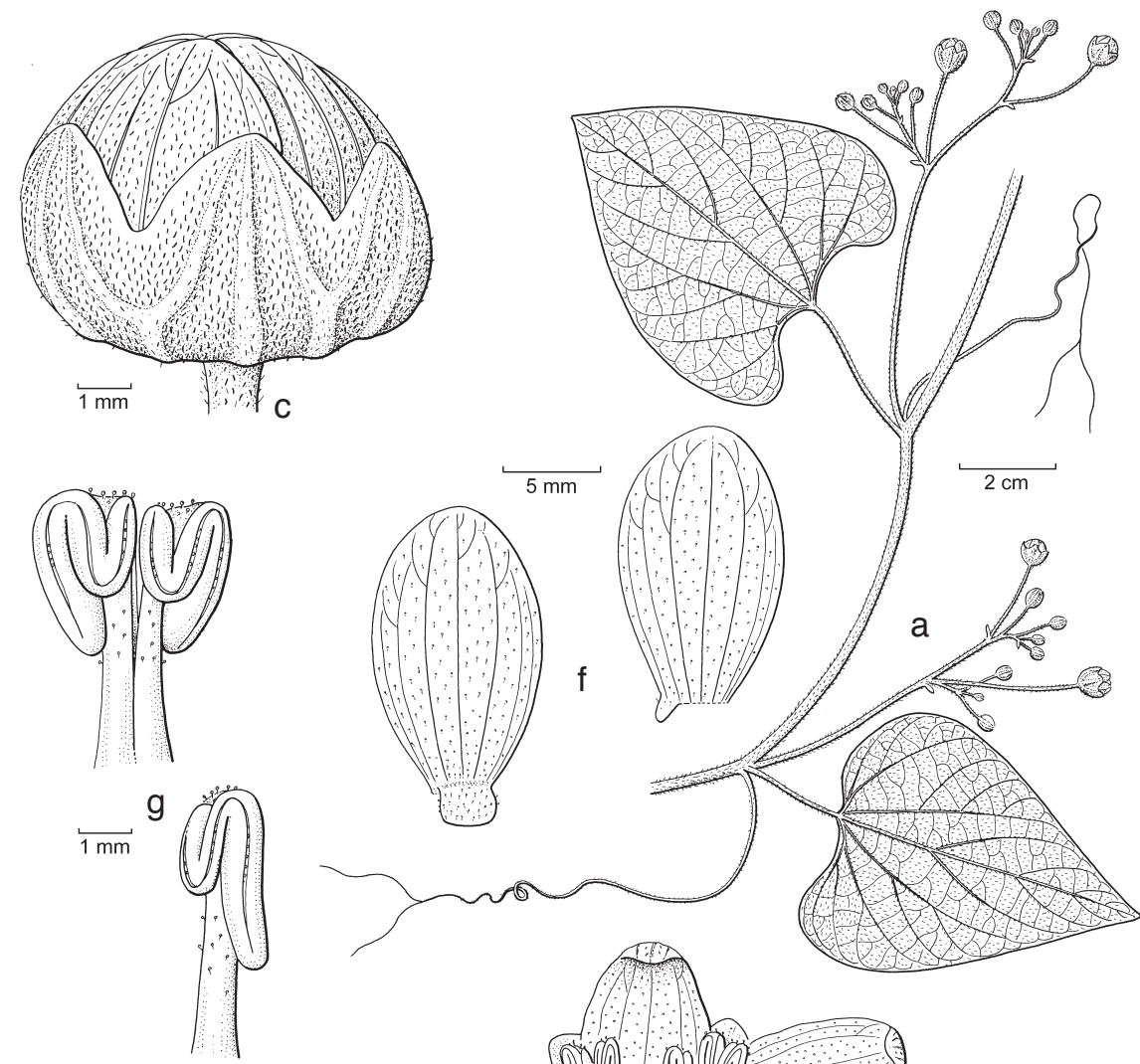
a. var. siamensis - Fig. 4a, b, 5

Male inflorescences simple, or once-branched, 10-20 cm long. Sepals of male flowers obtuse (rounded) or sub-acute at apex; petals broadly elliptic, apex rounded.

Field-notes - Mature fruits green. Lörzing 5595 gives the following observations (translated): "Climbing herb $8 \mathrm{~m}$, profusely branched; rare; male flowers: calyx bright red-brown, with bright green veins, when in flower the red colour vanishes for the greater part; corolla deeply 5-lobed, yellow, with pale yellow-green veins; anthers two with each 2 free thecae, and one with 1 theca and with two scale-like staminodia; always 1 stamen or staminodia basally adnate to a petal; stamens bright yellow or green-yellow, at the base yolk-yellow; 5 petals, all the same size, broadly obovoid, dorsally somewhat rounded; upper surface of leaves green, somewhat glossy, lower surface dull pale green; lower surface of young leaves chocolate with grey-green nerves."

Distribution - Possibly as the species, but see the note under var. silomaradjae.

Habitat \& Ecology - Open or disturbed places in evergreen forest, hill Dipterocarp forest on acid rock, forest edges, scrub; 0-1500 m altitude. Flowering: April; fruiting: May, September, November.

Note - The hairiness can be very different. Strongly long-haired is, e.g., Eberhardt 4273 (type of Momordica tonkinensis); almost glabrous is, e.g., Pételot 8414.

\section{b. var. silomaradjae (Merr.) W.J. de Wilde \& Duyfjes, stat. nov.}

Siraitia silomaradjae Merr. (1934) 200; A.M. Lu \& J.Q. Li in Li (1993) 54, f. 2. - Type: Bartlett $8702 a$ (holo US; iso L), male flowers in June 1927, Sumatra.

Male inflorescences long-peduncled, $15-25 \mathrm{~cm}$ long, (1 or) 2 (or 3) times branched panicles. Sepals of male flowers long-acute-acuminate, the apices in bud free and slightly out-curved; petals narrow, long-triangular, at base c. $4 \mathrm{~mm}$ wide, apex longacute (see Lu \& Li, 1993: f. 2).

Distribution - Only known from the type, Asahan area, northern Sumatra.

Notes -1 . The only other flowering collection of the species known from the Malesian area is Lörzing 5595 (BO, L) from Sibolangit, not far from Asahan. Although the flowers of Lörzing 5595 are in a bad state of conservation, they are more similar to those of var. siamensis than to those of var. silomaradjae.

2. We cannot decide on the distribution of var. silomaradjae beyond the type-locality as all other collections from Malesia (Sumatra, Peninsular Malaysia, Java, Borneo) are sterile, except one collection from Java in fruit.

\section{THLADIANTHA}

Thladiantha Bunge (1833) 29; Cogn. (1881) 421; (1916) 40; Gagnep. (1921) 1075; Craib (1931) 759; Chakrav. (1959) 125; Backer (1964) 296; Keraudren (1975) 29; C. Jeffrey (1980a) 27; A.M. Lu \& Zhi Y. Zhang (1981) 61; (1986b) 132; S.K. Chen (1995b) 297; J.Q. Li (1997) 103-127. - Type: Thladiantha dubia Bunge (China, near Beijing).

Herbaceous climbers, usually with tuberous rootstock; dioecious; stem and leaves without glandular hairs. Probract absent in sterile shoots, in inflorescences present or absent. Tendrils unbranched (all material from Thailand and Malesia) or 2-branched 

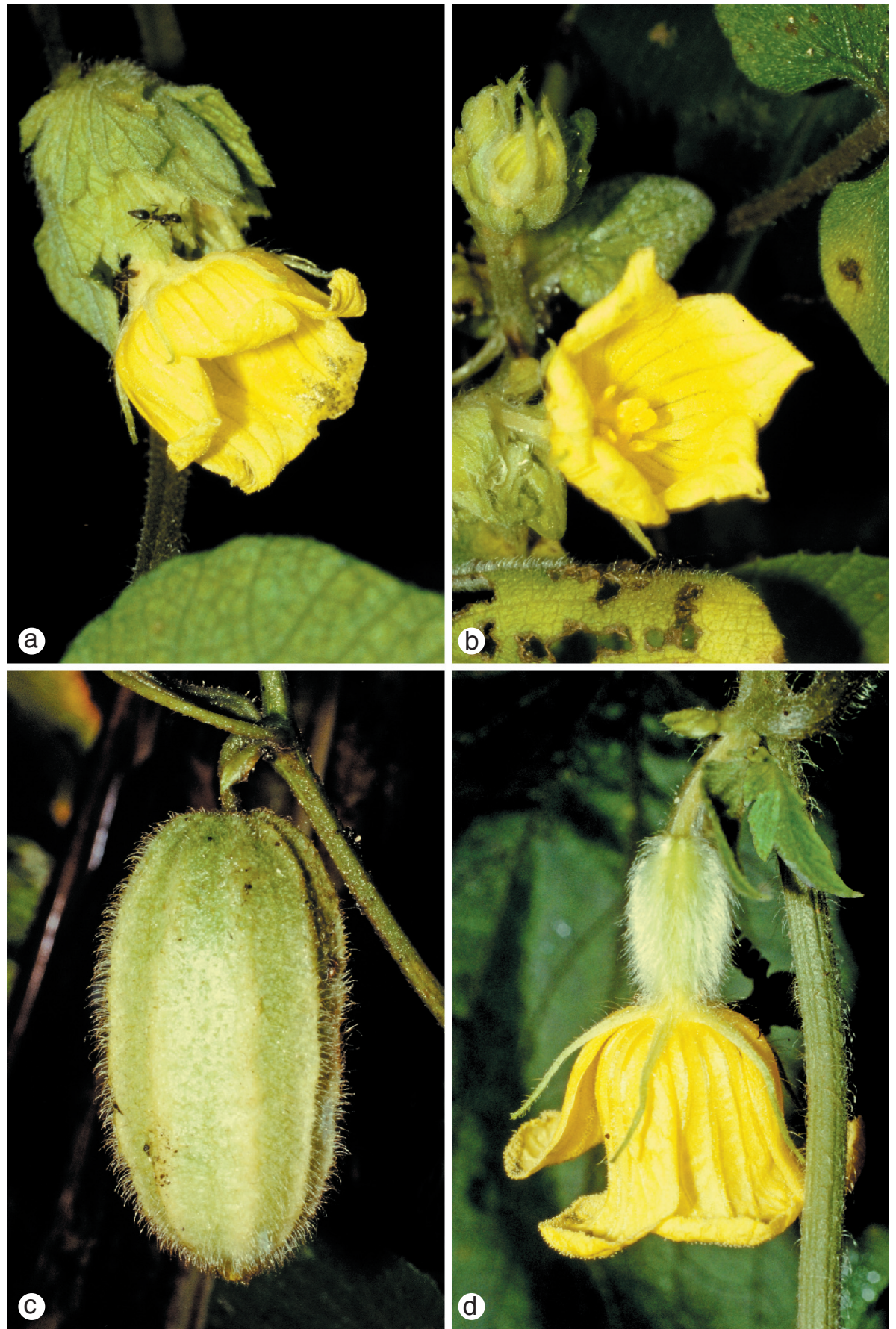

Plate 1. Thladiantha angustisepala W.J. de Wilde \& Duyfjes (a, b: Phonsena et al. 4637 (type); c: Phonsena et al. 5238; d: Phonsena et al. 5204). Photos De Wilde. 

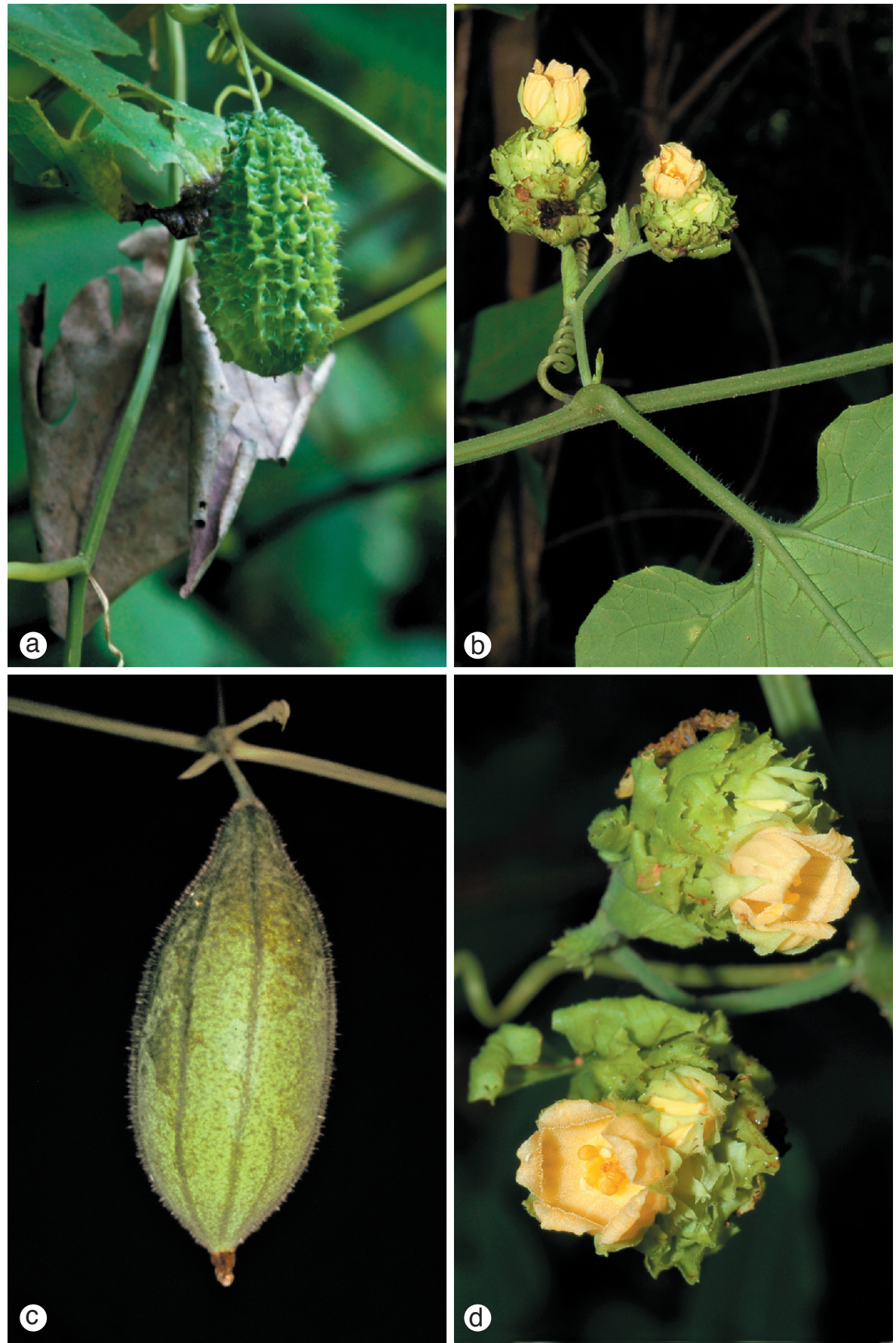

Plate 2. a. Thladiantha cordifolia (Blume) Cogn.; b-d. Sinobaijiania smitinandii W.J. de Wilde \& Duyfjes (a: photographed in Sumatra; b, d: Phonsena et al. (29-09-2006); c: Panyrat 131). Photos a: Haruo Katakura; b, d: Thamarat Putthai; c: Phornpithak Panyarat. 
and spiralling only above the point of branching. Leaf-blades ovate, usually simple, not lobed or palmately lobed, rarely 3-9-foliolate, green or brown on drying. Flowers yellow. Male flowers in racemes or panicles, rarely flowers solitary; receptacle-tube shallow, with eccentric half-globose basal disc; pedicels articulate towards apex; sepals linear to (narrowly) ovate; petals longer than sepals, (narrowly) ovate, obtuse, 1-3 with an adaxial basal scale; stamens (3-)5, inserted on or near throat of receptacle-tube, often in two pairs and one solitary, filaments long, thecae short, more or less straight, erect. Female flowers solitary or few in short racemes or panicles, similar to male flowers; stigmas subglobose; staminodes 5. Fruits (narrowly) ellipsoid, 2-6 cm long, fleshy, indehiscent, ribbed or verrucose, not sweet. Seeds numerous, ovoid or ellipsoid, finely sculptured, with or without narrow margin.

A genus of about 25 species distributed in SE Asia, extending SW to West Malesia (Sumatra, Java); 5 species in Thailand.

\section{KEY TO THE SPECIES OF THLADIANTHA IN THAILAND AND MALESIA}

1a. Leaf blade simple. Male bracts conspicuous, (0.5-)1-2 cm long. (Ovary and fruits \pm fenestrate, ribbed or verrucose: sect. Fidobractea A.M. Lu \& Zhi Y. Zhang

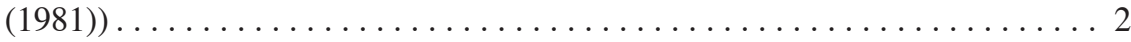

b. Leaf blade simple, not lobed or (deeply) lobed, or foliolate. Male bracts absent. (Ovary and fruits smooth, though hairy: sect. Thladiantha) . . . . . . . . 4

2a. Probract at base of male peduncle absent. Petiole and peduncle long-pilose. Plant

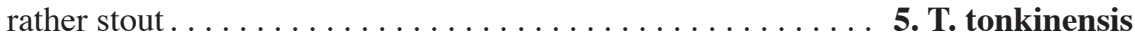

b. Probract absent or (its scar) present at base of male peduncle. Petiole and peduncle

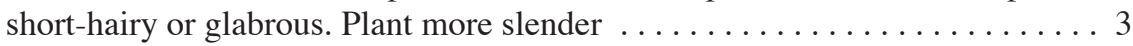

3a. Sepals linear, thread-like, less than $1 \mathrm{~mm}$ wide, 1 -veined. Fruit ribbed . . . . . . .

b. Sepals narrowly elliptic, $2-3 \mathrm{~mm}$ wide, 3 -veined. Fruit fenestrate . . . . . . .

2. T. cordifolia

4a. Plant (sub) glabrous. Leaves simple, lobed or not lobed, or foliolate 3. T. hookeri

b. Plant long-hairy. Leaves simple, not lobed ... . . . . . 4. T. indochinensis

1. Thladiantha angustisepala W.J. de Wilde \& Duyfjes, spec. nov. - Fig. 6e, f; Plate 1

Thladianthae cordifolia similis, sepalis angustis minus quam $1 \mathrm{~mm}$ latis, fructu laevi (non fenestrato) distinguenda. - Typus: Phonsena, De Wilde \& Duyfjes 4637, male (holo BKF; iso L), Thailand, Kaeng Krachan NP.

?Thladiantha cordifolia (Blume) Cogn. var. tomentosa A.M. Lu \& Zhi Y. Zhang (1981) 70. - Type: S.Q. Chen 12174 (PE, not seen).

Subligneous or herbaceous climber 4-10 m tall, putatively from a tuberous root; stem (leafy shoots) 2-3 mm diam., variously grey or yellowish brown soft long-hairy, hairs 1.5-4 mm long. Probract absent or rarely present at base of male peduncle, c. $10 \mathrm{~mm}$ long, basal part narrow, apex incised. Tendrils unbranched, spiralling over almost all their length, but basal part at least for $1 \mathrm{~cm}$ straight. Leaves: blade simple, (narrowly) ovate, $6-12(-15)$ by $4-9(-12) \mathrm{cm}$, base deeply cordate, apex acute-acuminate, margin 

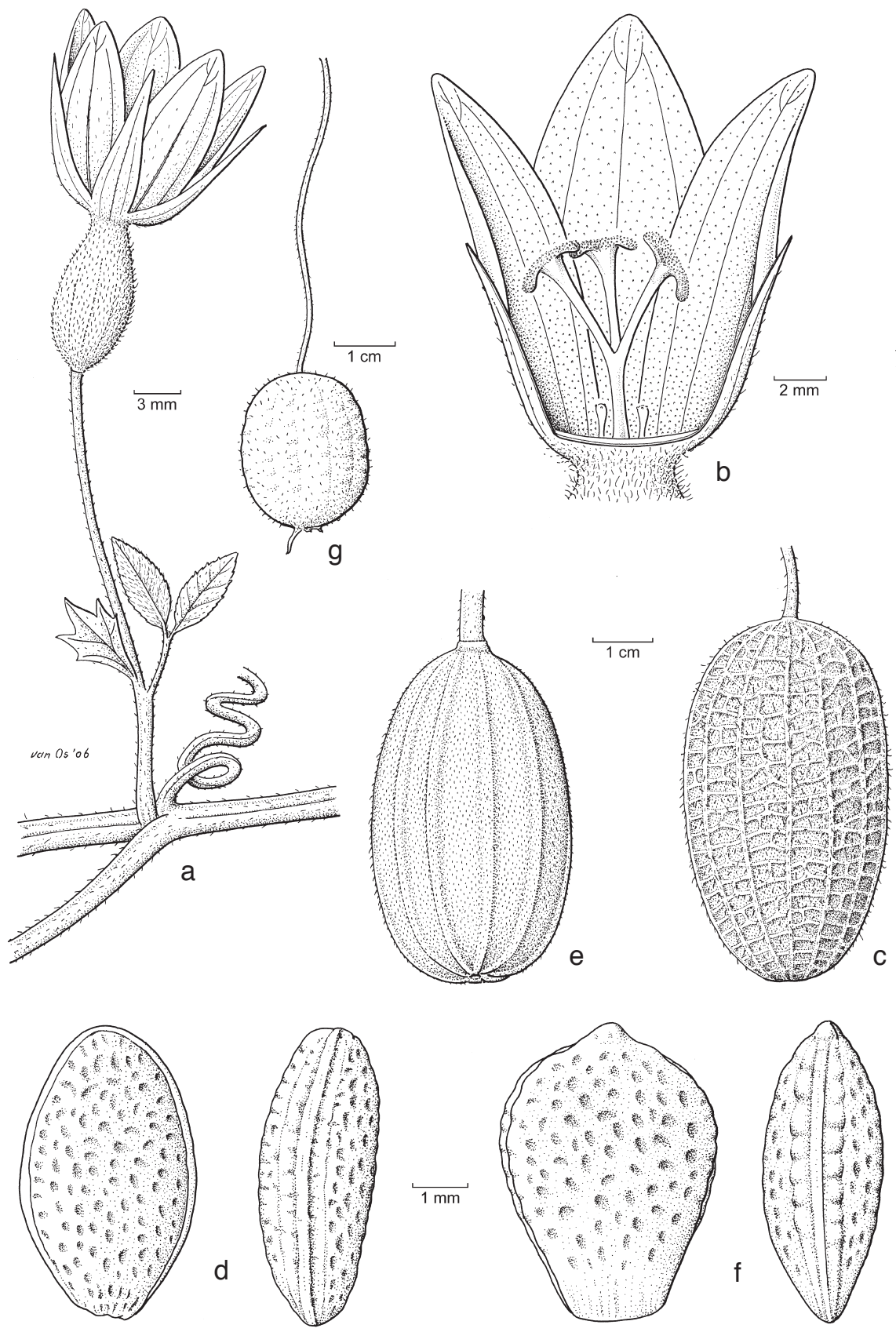

Fig. 6. a-d: Thladiantha cordifolia (Blume) Cogn. a. Node with 1-flowered female inflorescence; b. female flower, perianth partly removed, disc absent; c. fruit, note fenestrately pitted outer surface; d. seeds. - e, f: Thladiantha angustisepala W.J. de Wilde \& Duyfjes. e. Fruit; f. seeds. - g: Thladiantha tonkinensis Gagnep. Fruit (a: Docters van Leeuwen s.n. (28-09-1910); b: Wongprasert s.n. (SN 120885); c, d: Maxwell 99-96; e, f: Phonsena 3960; g: Balansa 4549). 
finely (coarsely) dentate, upper surface rather densely appressed scabrid-hairy, later on leaving cystoliths, lower surface densely softer hairy, especially on veins; petiole 2-9 cm long, (sparsely) soft-hairy. Male inflorescences: one (or rarely 2) peduncled raceme(s), sometimes with the persistent pedicel of a previously developed flower at base; peduncle 2-10 cm long; flowers few, bracteate, crowded, all pubescent with short or long hairs, hairs up to $2(-4) \mathrm{mm}$ long; bracts elliptic to narrowly elliptic or \pm wedge-shaped, $1-1.5$ by $0.4-1 \mathrm{~cm}$, shallowly or up to halfway incised, apices of lobes long-acute. Male flowers: pedicel 5-10 mm long, longer in basal solitary male flower; receptacle-tube cup-shaped, c. 5 by $6 \mathrm{~mm}$, throat minutely papillose; sepals linear, $6-10$ $\mathrm{mm}$ long, c. $1 \mathrm{~mm}$ wide or less, 1 -veined; petals (10-) 15 by $8-10 \mathrm{~mm}$, obtuse, inside papillose; filaments c. $4 \mathrm{~mm}$ long, anthers (2-) 2.5 by c. $1.5 \mathrm{~mm}$; petal basal scales papillose, largest c. 3 by $2 \mathrm{~mm}$, and 3 (or 4) much smaller ones; disc subglobose, c. $2 \mathrm{~mm}$ diameter. Female flower: solitary (or 2 or 3 in a short bracteate raceme); pedicel $5(-10)$ $\mathrm{mm}$ long; perianth as in male flowers but somewhat larger; ovary ellipsoid, c. 12 by 7 $\mathrm{mm}$, faintly 10-ribbed, densely (appressed) hairy, hairs c. $2 \mathrm{~mm}$ long; receptacle-tube narrow; sepals (10-) $15 \mathrm{~mm}$ long, linear; petals $20-22$ by $15 \mathrm{~mm}$; style-column c. 0.5 $\mathrm{mm}$ long, arms 4(-5) mm long, stigmas irregularly broadly reniform, papillose, each 3-4 mm wide; petal basal scales 3, c. $1 \mathrm{~mm}$ long; staminodes 3(-5), erect, 1-1.5 mm long, papillose. Fruit solitary (or 2), ellipsoid, $4-5$ by $2.5-3 \mathrm{~cm}, 10$-ribbed, hairy, smooth, not fenestrately ornamented between the ribs; fruiting pedicel $1-1.5 \mathrm{~cm}$ long. Seeds ovoid, c. 4 by 3 by $2 \mathrm{~mm}$, pale brown, finely rugose.

Field-note - Male corollas falling off in late morning.

Distribution - Thailand (N, SW), Laos.

Habitat \& Ecology - Scrub and seasonal forest edges; 300-1500 m altitude. Flowering: February to September; fruiting: September, October.

Note - The collections Kerr 2449 and Maxwell 05-138, both from Chiang Mai Province, are aberrantly densely long-haired in all parts.

Specimens examined:

THAILAND. Kerr 2449 (male); Larsen et al. 46169 (male); Maxwell 05-138 (male, specimen very hairy); Parnell et al. 95-516 (male); Phonsena et al. 3960 (female, fruit, spirit); 3961 (male); 3964 (male); 4636 (female, fruit); 4637 (type, male); 4680 (male); 4681 (female); Pooma 1253 (male); Van Beusekom et al. 4652 (male); Wongprasert 997-34 (male?).

LAOS. D'Alleizette s.n. (male, possible same as Poilane 26355); Poilane 26355 (male); Poilane s.n. (male).

\section{Thladiantha cordifolia (Blume) Cogn. - Fig. 6a-d, 7; Plate 2a}

Thladiantha cordifolia (Blume) Cogn. (1881) 424; (1916) 51; C. Jeffrey \& Kashyapa (1960) 461-463; Backer (1964) 296; Keraudren (1975) 30, p.p.; C. Jeffrey (1980a) 30; A.M. Lu \& Zhi Y. Zhang (1986b) 135, f. 37: 13, 14, p.p.; S.K. Chen (1995b) 300, p.p. - Luffa cordifolia Blume (1826) 929; Ser. (1828) 302; M. Roem. (1846) 63; Miq. (1856) 666. - Lectotype (here chosen): Blume 1464, fruit, barcode L0001624 (holo L; iso L), Java.

Thladiantha cordifolia (Blume) Cogn. forma glabrescens Hochr. (1934) 287. - Type: Hochreutiner 1255 (iso L), Java.

Thladiantha calcarata Cogn. (1880) Errata, without page; (1881) 423; (1916) 50; Gagnep. (1921) 1076, p.p.; Craib (1931) 759; Chakrav. (1959) 125. - Lectotype (Jeffrey, 1980b: 790): Wallich Cat. 6740A (K-W) ('Momordica calcarata'), collection Gomez, Sylhet.

Trichosanthes javanica Miq. (1856) 678. - Type: Junghuhn 875 (iso L), Java.

Gymnopetalum piperifolium Miq. (1856) 680. - Type: Horsfield s.n. (K, not seen), Java. 
Gymnopetalum horsfieldii Miq. (1856) 680. - Type: Horsfield s.n. (K, not seen), Java.

Thladiantha calcarata Cogn. var. subglabra Cogn. (1881) 424. - Type: Hooker \& Thomson 2057 (iso K), Khasia, India.

Thladiantha dubia auct. non Bunge: Hook. (1864) t. 5469, p.p., for the female flower and fruit; C.B. Clarke (1879) 631 (see Cogniaux, Errata (1880) under Thladiantha calcarata).

Perennial much-branched climber to $8 \mathrm{~m}$ long; subglabrous or hairy; stem of leafy shoots 2(-3) mm diameter. Probract usually present. Tendrils unbranched, spiralling either throughout their length or only in the upper part. Leaves: blade simple, ovate, 6-10 by 4-9 $\mathrm{cm}$, deeply cordate, apex acute-acuminate, margin coarsely or finely dentate, upper surface appressed-hairy, scabrous by cystoliths, lower surface hairy or subglabrous; petiole 3-6 cm long, finely hairy or subglabrous. Male inflorescences: a peduncled bracteate raceme (sometimes with a previously developed solitary flower at base), 5-10 cm long, sparsely minutely pubescent; peduncle $2-5 \mathrm{~cm}$ long, at or close to the base with a rhombiform or sub-elliptic (5-)10 mm long irregularly incised probract; flowers few or several, bracts conspicuous, close together, or in older inflorescences the scars of the fallen flowers and bracts more spaced, forming an up to $3 \mathrm{~cm}$ long spike, with few bracteate flowers and buds at apex; bracts obovate or obtriangular, 10-15 mm long, the margin in the apical half (irregularly) dentate or incised to c. 1/4 deep. Male flowers: pedicel c. $10 \mathrm{~mm}$ long; receptacle-tube obliquely cup-shaped, tapering, 3-4 mm diam., throat short-hairy; sepals long-triangular, or narrowly elliptic, $8-12$ by $3-5$ $\mathrm{mm}$, acute, 3(-5-)veined, sometimes reflexed; petals in bud erect and much shorter than sepals, expanded petals obovate-elliptic, $15-20$ by c. $15 \mathrm{~mm}$, apex rounded and faintly dentate; filaments short, 3-5 mm long, \pm curved, \pm dilated towards the base, anthers 3-5 mm long, slightly curved, median(?) petal with conspicuous curved scale, c. $4 \mathrm{~mm}$ long, concealing the disc in the receptacle-tube; disc inserted slightly laterally, large, ellipsoid, c. $4 \mathrm{~mm}$ long. Female flowers: solitary; pedicel 10-40 mm long, with probract at or near base; perianth as in male flowers; ovary ovoid-ellipsoid, densely woolly grey-hairy, c. $10 \mathrm{~mm}$ long; style-column c. $2 \mathrm{~mm}$ long, arms 4-5 mm long, stigmas 2-lobed, lobes broadly reniform, each c. $3 \mathrm{~mm}$ diam.; staminodes c. $1.5 \mathrm{~mm}$ long, erect. Fruit solitary; narrowly ellipsoid, (2-)3-6 by $2-2.5 \mathrm{~cm}$, broadly rounded at both ends, sparsely or densely bristly hairy, outer surface fenestrately pitted in c. 15 rows; fruiting pedicel $1.5-4 \mathrm{~cm}$ long. Seeds ovoid, 5-6 mm long, somewhat flattened, rugose, margin shallowly 2 -grooved.

Field-note - The seeds are embedded in whitish pulp. Fruits are edible (Maxwell 99-96).

Distribution - [Eastern?] E India, Myanmar, Thailand (N, SW) to S China, extending to Sumatra and Java (type). Not recorded from Peninsular Thailand and Peninsular Malaysia.

Habitat \& Ecology - Seasonal rainforest, disturbed hill Dipterocarp forest along trail, also open areas and degraded forest with much bamboo, often along stream; sandy soil, shale bedrock, and limestone; 200-800 m altitude. Flowering: April, May, August; fruiting: August.

Note - Thladiantha cordifolia belongs with two other here treated species, T. tonkinensis and T. angustisepala to the section Fidobractea A.M. Lu \& Zhi Y. Zhang (1981) of which the type species is Thladiantha cordifolia. The section is characterized by the male flowers arranged in a bracteate raceme, and by the imbricately arranged, large, flabellate bracts with an incised-dentate margin. In the section belong furthermore three 
Chinese species: 1) T. globicarpa A.M. Lu \& Zhi Y. Zhang (1981) (C China), similar to $T$. angustisepala, also with narrow sepals but differing in a more slender habit, and smaller fruit; 2) T. grandisepala A.M. Lu \& Zhi Y. Zhang (1981) (Yunnan); and 3) T. medogensis A.M. Lu \& J.Q. Li in Li et al. (1992) (Xizang), the latter two species have much broader sepals.
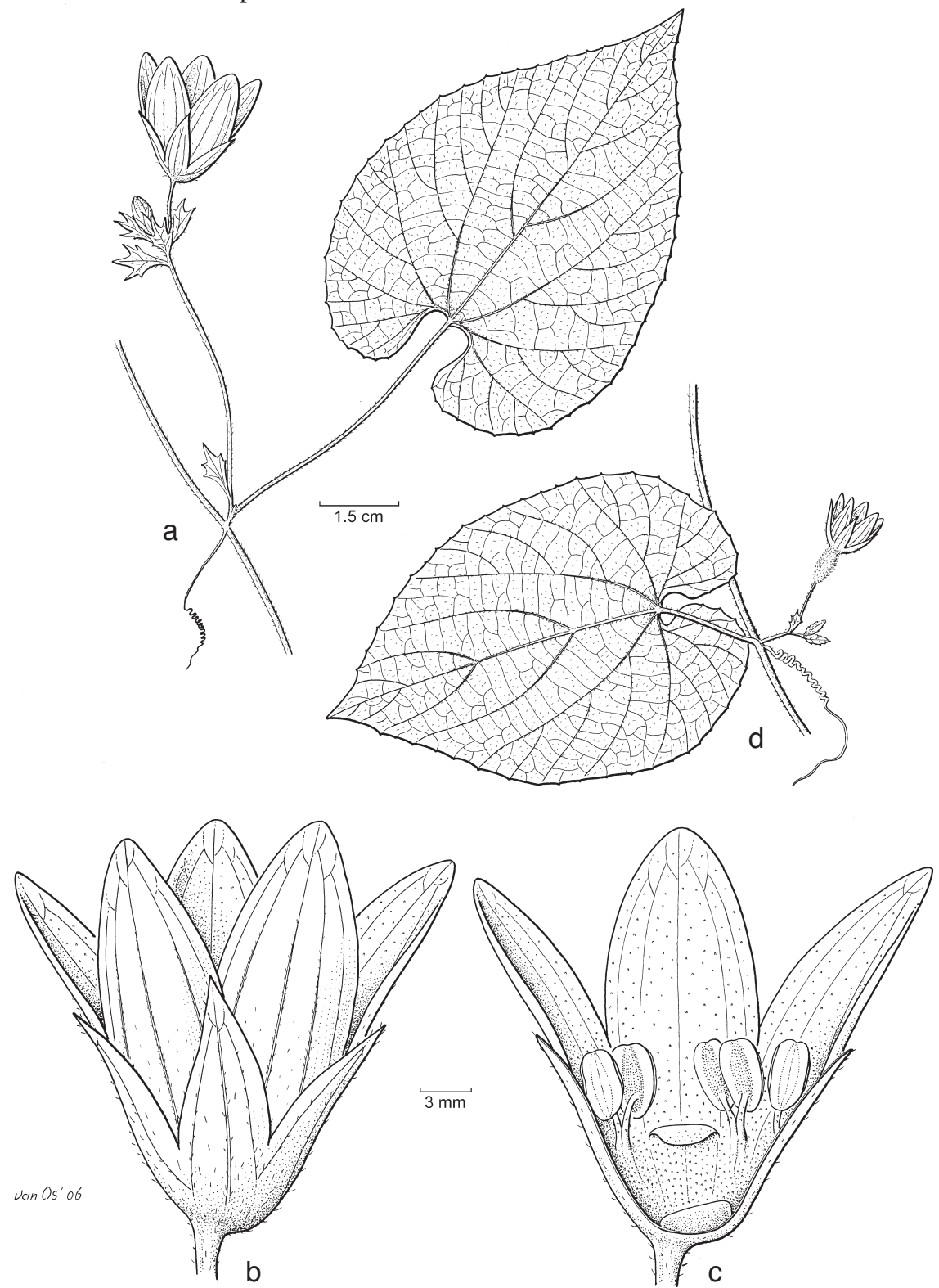

Fig. 7. Thladiantha cordifolia (Blume) Cogn. a. Node with male inflorescence; b. male flower; c. idem, laid open, partly schematic, note eccentrically situated disc-gland; d. node with 1-flowered female inflorescence (a-c: Wieringa \& Janzen 3417; d: Docters van Leeuwen s.n. (28-09-1910)). 


\section{Thladiantha hookeri C.B. Clarke - Fig. 8}

Thladiantha hookeri C.B. Clarke (1879) 631; Cogn. (1881) 425; (1916) 52; Gagnep. (1921) 1078; Craib (1931) 759; Chakrav. (1946) 64 (not seen); (1959) 127, incl. variety and forms; Keraudren (1975) 32, f. 6: 7-9; C. Jeffrey (1980a) 35; A.M. Lu \& Zhi Y. Zhang (1986b) 149; S. K. Chen (1995b) 305. - Lectotype (here chosen): Hooker \& Thomson 1703 (K), Khasia.

Hemsleya? tonkinensis Cogn. (1893) 613. - Type: Balansa 4011 (G, not seen), Tonkin.

Hemsleya trifoliolata Cogn. (1909) 304. - Thladiantha trifoliolata (Cogn.) Merr. (1937) 261. - Type: Henry $12295 D$ (Z, not seen), Yunnan.

Thladiantha pentadactyla Cogn. (1916) 52. - Thladiantha hookeri C.B. Clarke var. pentadactyla (Cogn.) A.M. Lu \& Zhi Y. Zhang (1981) 80. - Type: Henry 12295D (B†, not seen), Yunnan.

Thladiantha heptadactyla Cogn. (1916) 52; Gagnep. (1918a) 290. - Thladiantha hookeri C.B. Clarke var. heptadactyla (Cogn.) A.M. Lu \& Zhi Y. Zhang (1981) 81. - Type: Delavay s.n. (P), Yunnan, Lou Kong.

Thladiantha digitata H. Lév. (1916) 65. - Type: Maire (E.E.M.) s.n. (E), Yunnan.

Hemsleya yunnanensis Cogn. (1916) 27. - Type: Henry 9057 (B†, not seen), Yunnan.

Herbaceous few-branched climber 1.5-4 m long, from a tuberous root; young parts finely hairy, largely glabrescent; stem 2(-4) mm diameter. Probract absent, except in connection with inflorescences, in male small, up to $5 \mathrm{~mm}$ long, at or towards base of inflorescences, in female not obvious. Tendrils unbranched, not spiralling basally. Leaves: blade simple, not lobed or lobed, or 3- or 5-foliolate, (broadly) ovate in outline, $7-20(-25)$ by $4-15(-20) \mathrm{cm}$, base deeply cordate, apex acute-acuminate, margin finely (coarsely) dentate; upper surface with sparse (minute) coarse hairs, later on leaving sparse cystoliths, lower surface glabrous, except for few hairs on veins; petiolules to $10 \mathrm{~mm}$ long; petiole $1.5-7 \mathrm{~cm}$ long. Male inflorescences: usually a peduncled raceme (rarely few-branched, see note 1), 2-12 cm long, including 1.5-5 $\mathrm{cm}$ long peduncle, early glabrescent, at base usually with a scar of fallen small probract, and a solitary pedicel 20-30 mm long of previously developed flower; flowers in raceme (few to) numerous; lower portion of pedicels persistent; bracts absent. Male flowers: buds with open calyx, imitating small flowers; pedicels 5-15 $\mathrm{mm}$ long; receptacle-tube shallowly cup-shaped, 2-3 by 5-7 mm, slightly oblique at base because of eccentric disc, inside minutely gland-hairy, throat minutely gland-hairy; sepals narrowly elliptic, 4-6 by 2-2.5 mm, 3- or 5-veined, \pm out-curved, apex greenish (often black on drying), (sub)acute; petals ovate to narrowly ovate, $10-15$ by $8-10 \mathrm{~mm}$, obtuse, $5(-7)$-veined, at apex of midvein with a minute gland; filaments short, 3-4 mm long, anthers c. 2.5 $\mathrm{mm}$ long; median? petal at base with a large curved scale, c. $2.5 \mathrm{~mm}$ long, concealing the disc; disc globose-ellipsoid, c. $2.5 \mathrm{~mm}$ diameter. Female flowers: solitary or 2 or 3 in up to $2 \mathrm{~cm}$ long raceme; pedicel (10-) $20 \mathrm{~mm}$ long (but see note 2); sepals and petals similar to those of male flowers, but narrower; ovary subfusiform, c. 15 by $3 \mathrm{~mm}$, at apex long-tapering, brownish short-woolly hairy; style c. $2.5 \mathrm{~mm}$ long, stigma-arms $0.5(-1) \mathrm{mm}$ long, stigmas reniform, c. $1.5 \mathrm{~mm}$ diam.; staminodes in two pairs and 1 solitary, c. $0.5 \mathrm{~mm}$ long, glandular-papillose; basal petal scale not obvious. Fruit (according to Lu \& Zhang, 1986b): (narrowly) ellipsoid, $4-6$ by $2-3 \mathrm{~cm}$, rounded at both ends, smooth. Seeds broadly ovoid, $6-7$ by $5 \mathrm{~mm}$, base obtuse-rounded, smooth.

Field-note - The leaves are variable in shape, simple or trifoliolate, often on the same twig. The inner side of the corolla is darker yellow than the outer side.

Distribution - NE India (type), Bhutan, S China (Yunnan, Guizhou), Myanmar, Thailand (N, SW), N Vietnam; (from Laos no collections seen). 
Habitat \& Ecology - Edges and scrub of evergreen forest, deciduous hardwood forest; on granite and shale as well as on limestone outcrop and rugged limestone terrain; 200-1400 m altitude. Flowering: May to November; no fruiting specimens known from Thailand.

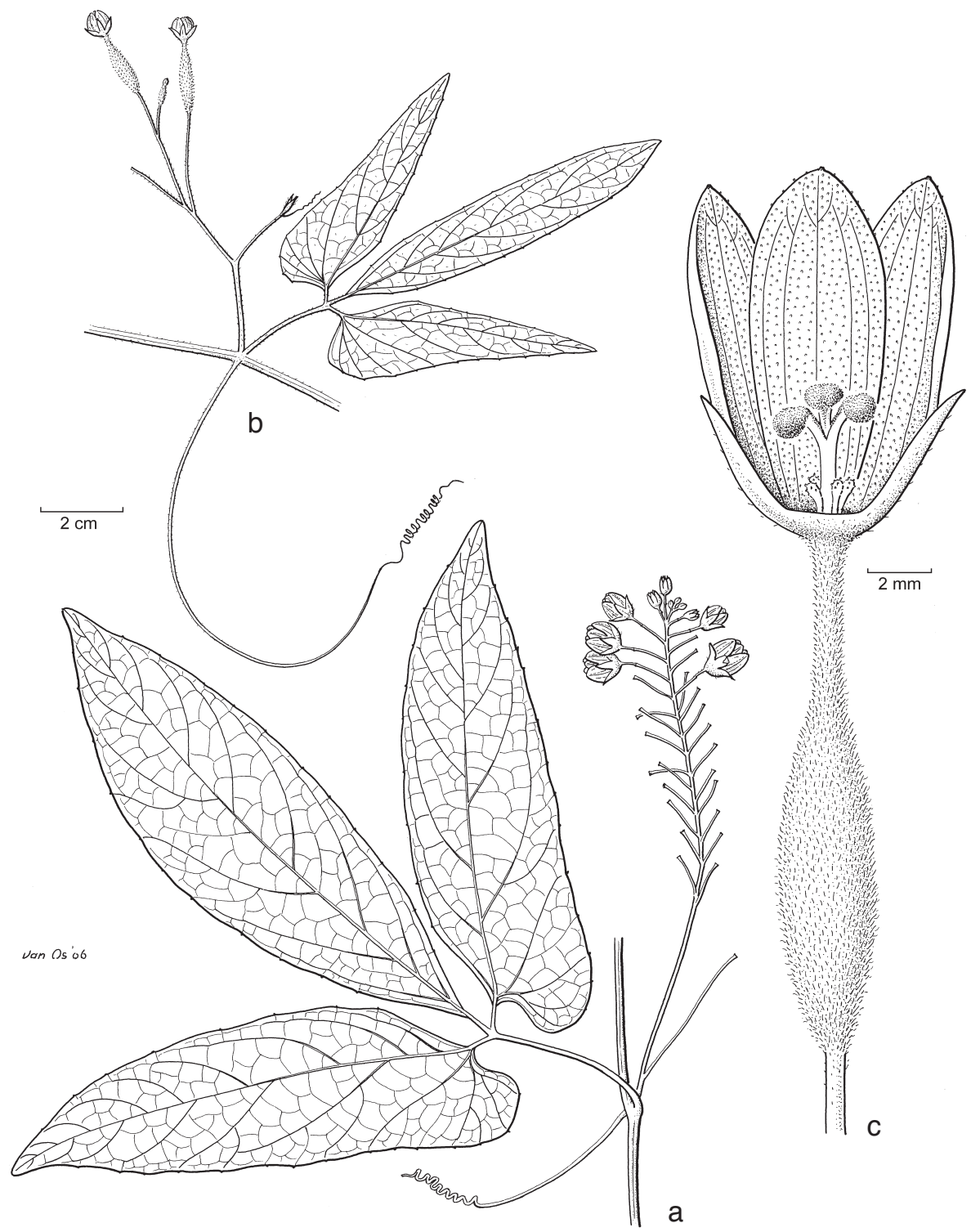

Fig. 8. Thladiantha hookeri C.B. Clarke. a. Leafy node with male inflorescence, note persistent pedicels; b. leafy node with female inflorescence; c. female flower, opened (a: Maxwell 90-860; b, c: Sai Jai 21). 
Notes - 1. Branched male inflorescences can be interpreted as a short lateral branch without leaves and tendrils; the solitary flower is lacking at the base of such compound inflorescence.

2. Material in P indicate that the status of T. pentadactyla Cogn. and T. heptadactyla Cogn., both from China, with foliolate leaves and petioluled leaflets and long-pedicelled female flowers, as ordinary synonyms of $T$. hookeri need further study. Solitary female flowers in T. heptadactyla have up to $10 \mathrm{~cm}$ long pedicels.

\section{Thladiantha indochinensis Merr.}

Thladiantha indochinensis Merr. (1940) 386; Keraudren (1975) 33. - Lectotype (Jeffrey, 1980a): Pételot $2194(\mathrm{GH}), \mathrm{N}$ Vietnam, Chapa.

Climber a few meters long, putatively with a tuberous rootstock; leafy shoot 2-3 mm thick, soft grey-hairy, hairs 1-2 mm long, glandular or not. Probract not obvious. Tendrils unbranched, not spiralling in the basal third. Leaves: blade simple, ovate or narrowly ovate, $9-17$ by $5-10 \mathrm{~cm}$, base deeply cordate, apex acute-acuminate, margin finely dentate, upper surface sparsely hairy, cystoliths not obvious, lower surface more densely hairy, especially on veins; petiole $2.5-6 \mathrm{~cm}$ long, soft-hairy. Male inflorescences: flowers solitary or in an up to 12 -flowered peduncled raceme to $10 \mathrm{~cm}$ long; bracts absent (possibly minute and early caducous); peduncle to $5 \mathrm{~cm}$ long, hairy. Male flowers: pedicel 15-25 mm long, hairy; receptacle-tube broadly campanulate, c. 3 by 4-5 mm, outside with long, stiff, whitish hairs, throat finely papillose-hairy; sepals narrowly ovate, c. $5 \mathrm{~mm}$ long, at base $1.5(-2) \mathrm{mm}$ wide, acute, hairy; petals ovate to narrowly ovate, $15-20$ by 7-10 $\mathrm{mm}$, obtuse, 5-7-veined, inside and margin papillose; filaments 3.5-4 mm long, papillose, anthers 3-3.5 mm long; petal basal scales: 1 large, c. $3 \mathrm{~mm}$ long, with 2 or 3 smaller ones forming a finely hairy diaphragm-like ring, c. $0.5 \mathrm{~mm}$ high; disc 1-1.5 mm across. Female flowers: solitary; pedicel (5-)10 $\mathrm{mm}$ long; perianth smaller than in male flowers, petals narrower; ovary short-fusiform, c. 4 by 2.5-3 mm, subglabrous or sparsely hairy; sepals c. $4 \mathrm{~mm}$ long; petals c. 13 mm long; style-column c. $2.5 \mathrm{~mm}$ long, style-arms (1.5-) $2 \mathrm{~mm}$ long, stigmas c. $1 \mathrm{~mm}$ diam.; staminodes in two pairs and 1 solitary, erect, c. $0.5 \mathrm{~mm}$ long, papillose; petal basal scale small. Fruit not known.

Distribution - Thailand (N), Vietnam (type).

Habitat \& Ecology - Open forest; 1500-1800 m altitude. Flowering: August, September.

Note - Jeffrey (1980a: 29) identified the specimens (Pételot 2185, 2194) on which Thladiantha indochinensis Merr. was based as T. nudiflora Forbes \& Hemsl., a species from China, this identification was confirmed by Zhi Y. Zhang in 1990 on an identification slip (A). In our opinion the Thai collection Vidal 5175 (male and female), of which the male flowers are solitary or few together, is similar to the type of $T$. indochinensis and these specimens are sufficiently different to keep the species separate. Thladiantha nudiflora has 2-branched tendrils and its ovary is described as narrowly oblong, $12-15$ by $4-5 \mathrm{~mm}$ (in T. indochinensis the ovary is short-fusiform, c. 4 by $2.5-3 \mathrm{~mm})$.

Specimens examined:

Pételot 2185, 2194, both N Vietnam, Chapa; Vidal 5175, Doi Chiang Dao (P, male; BKF, female; AAU?). 


\section{Thladiantha tonkinensis Gagnep. - Fig. $6 \mathrm{~g}$}

Thladiantha tonkinensis Gagnep (1918a) 292; (1921) 1078. - Type: Balansa 4003 (holo P; iso P), North Vietnam, Tonkin, Vallée de Langkok, Mt Bavi.

Thladiantha calcarata Cogn. var. tonkinensis Cogn. (1916) 50. - Lectotype (here chosen): Balansa 4004 (holo P; iso E, P).

Thladiantha calcarata auct. non C.B. Clarke: Gagnep. (1918a) 293, in clavis; (1921) 1076, f. 122.

Thladiantha cordifolia auct. non (Blume) Cogn.: Keraudren (1975) 30, f. 6: 1-6; S. K. Chen (1995b) 300.

Climber to $10 \mathrm{~m}$ tall, possibly with tuberous root; stem of leafy shoots (2-)3(-4) mm thick, rather densely soft-patently hairy, hairs pale brown, (1-)2-3 mm long. Probract usually absent, occasionally present (when male inflorescence with solitary flower at base of peduncle present), \pm rhomboid-elliptic, c. $1.5 \mathrm{~cm}$ long, dentate or incised in apical half. Tendrils unbranched, not spiralling in lower third, hairy. Leaves: blade simple, ovate (or narrowly ovate), 7-17 by $4.5-12 \mathrm{~cm}$, base deeply cordate, apex acuteacuminate, margin (densely) finely dentate, upper surface \pm sparsely mixed-hairy, the shorter hairs finally leaving small cystoliths, lower surface more densely hairy especially on veins; petiole 4-12 cm long, soft-hairy. Male inflorescences: a peduncled raceme, $6-15 \mathrm{~cm}$ long, with up to 20 bracteate flowers crowded in a head $2.5-4 \mathrm{~cm}$ diam. at apex; peduncle 5-10 cm long, soft-hairy, hairs (1-)2-3 mm long; bracts \pm rhomboidobovate or wedge-shaped, $1.5(-2)$ by $1.5 \mathrm{~cm}$, the apical part \pm rounded, shallowly or deeply (1/4 to $1 / 2)$ incised, the lobes acute to long-acuminate, base narrow, subglabrous to (sparsely) long-hairy, hairs $0.5-2 \mathrm{~mm}$ long; bracts persistent after flowering. Male flowers: sparsely hairy, frequently up to 3 open flowers in one raceme; pedicel short, $2(-3) \mathrm{mm}$ long, articulate towards base; receptacle-tube broadly cup-shaped, c. 3 by $5 \mathrm{~mm}$, finely papillose in the throat; sepals (narrowly ovate-) linear, $8-12$ by $1-1.5$ $\mathrm{mm}, 3(-5-)$ nerved, sparsely hairy; petals narrowly ovate, obtuse, $15-20$ by $7-10$ $\mathrm{mm}, 7$-nerved, inside finely papillose; basal petal scales: one or two large, 2-2.5 mm long, up to 3 smaller ones present or absent; filaments 3-4 mm long (one shorter than the others), glandular-hairy, anthers 3-4 mm long; disc c. $2.5 \mathrm{~mm}$ diameter. Female flowers: not known. Fruit solitary, subglobose, c. 3 by $2.5 \mathrm{~cm}$, irregularly striateverrucose, (sparsely) hairy; fruiting pedicel c. $6 \mathrm{~cm}$ long. Seeds pear-shaped (or ovoid), somewhat narrowed at base, rather flattened, c. 5 by 3.5 by $1.5 \mathrm{~mm}$, finely verrucose, margin \pm square.

Field note - Fruit fleshy, dirty white.

Distribution - S Yunnan, N Thailand, N Vietnam (type), Laos.

Habitat \& Ecology - Hill evergreen forest, scrub edges by streams; 300-1500 m altitude. Flowering: all year round; fruiting: July.

Note - Thladiantha calcarata C.B. Clarke var. tonkinensis Cogn. (1916: 50) was based on Balansa $4004 \& 4549$ and described as a rather glabrescent plant. We have examined Balansa 4004 in P and E and Balansa 4549 in L. The latter is rather hairy, the former quite glabrous. Therefore, we have chosen Balansa 4004 as lectotype.

\section{ACKNOWLEDGEMENTS}

We thank the curators of AAU, BK, BKF, BO, CMU, E, GH, K, KEP, L, M, MO, P, QBG, TCD, W, and WAG, who made their collections available for this study. Hospitality was received in BKF, E, 
P, TCD, and W. Indispensable fieldwork was organized for us by Kongkanda Chayamarit (BKF); it was carried out together with our friend Phongsak Phonsena (BKF). It was a pleasure to co-operate with Hanno Schäfer (M). We thank Haruo Katakura (SAP), Thamarat Putthai \& Phornpithak Panyarat (both BKF), for providing informative photos. Walter Kittredge (A) scanned some specimens of Thladiantha for our study in Leiden. Jan-Frits Veldkamp (L) translated the descriptions of the new taxa into Latin. Jan van Os (L) prepared the beautiful drawings. Ben Kieft $(\mathrm{L})$ scanned the drawings for publication; he also provided the digital imaging of the photos. Luc Willemse (L) helped with the realization of the identification list, using BRAHMS.

\section{REFERENCES}

Backer, C.A. 1964. Cucurbitaceae. In: C.A. Backer \& R.C. Bakhuizen van den Brink Jr., Flora of Java 1: 292-307. Noordhoff, Groningen.

Blume, C.L. 1826. Bijdragen tot de Flora van Nederlandsch Indië 15: 922-940. Ter Lands Drukkerij, Batavia.

Chakravarty, H.L. 1946. Studies on Indian Cucurbitaceae; with special remarks on distribution and uses of economic species. Indian J. Agric. Sci. 16: 1-90, (not seen).

Chakravarty, H.L. 1952 New finds of Indian Cucurbitaceae. J. Bombay Nat. Hist. Soc. 50: 894901.

Chakravarty, H.L. 1959. Monograph on Indian Cucurbitaceae. Rec. Bot. Surv. India 17: 1-234.

Chen, S.K. 1995a. Siraitia. In: C.Y. Wu, C. Chen \& S.K. Chen (eds.), Fl. Yunnan. 6:310-314. Science Press, Beijing.

Chen, S.K. 1995b. Thladiantha. In: C.Y. Wu, C. Chen \& S.K. Chen (eds.), Fl. Yunnan. 6: 297-310. Science Press, Beijing.

Clarke, C.B. 1879. Cucurbitaceae. In: J.D. Hooker, Flora of British India 2: 604-635. Reeve \& Co., London.

Cogniaux, C.A. 1880. Errata to vol. 2, part 3. In: J.D. Hooker, Flora of British India 3, 7: without page. Reeve \& Co., London.

Cogniaux, C.A. 1881. Cucurbitaceae. In: A. \& C. de Candolle, Monographiae Phanerogamarum Prodromi 3: 325-951.

Cogniaux, C.A. 1893. Le genre Siolmatra H. Baill. et la tribu des Zanoniées. Bull. Herb. Boissier 1: 609-613.

Cogniaux, C.A. 1909. Hemsleya nova Chinensis (H. trifoliata). Fedde, Repert. Nov. Sp. 6: 304.

Cogniaux, C.A. 1916. Cucurbitaceae-Fevilleae et Melothrieae. In: A. Engler, Pflanzenreich 66, iv.275.1: 1-277. Engelmann, Leipzig.

Craib, W.G. 1914. Contributions to the Flora of Siam. Additamenta 5. Bull. Misc. Inform. Kew: $4-11$.

Craib, W.G. 1931. Cucurbitaceae. Flora Siamensis Enumeratio. A list of the plants known from Siam 1: 750-770. Siam Society, Bangkok.

De Wilde, W.J.J.O. \& B.E.E. Duyfjes. 2002. Synopsis of Momordica (Cucurbitaceae) in SE Asia and Malesia. Bot. Zhurn. (St. Petersburg) 87: 132-148.

De Wilde, W.J.J.O. \& B.E.E. Duyfjes. 2003. The genus Baijiania (Cucurbitaceae). Blumea 48: $279-284$.

Gagnepain, F. 1918a. Révision des Thladiantha asiatiques du Muséum. Bull. Mus. Natl. Hist. Nat. 24: $287-296$.

Gagnepain, F. 1918b. Cucurbitacées nouvelles de l'Herbier du Muséum. Bull. Mus. Natl. Hist. Nat. 24: $371-380$.

Gagnepain, F. 1921. Cucurbitacées. Fl. Indo-Chine 2: 1030-1095. Masson \& Cie., Paris.

Hayata, B. 1911. Materials for a Flora of Formosa. J. Coll. Sci. Imp. Univ. Tokyo 44: 117-122.

Hochreutiner, B.P.G. 1934. Plantae Hochreutineranae. Fascicule 3. Candollea 5: 287-290.

Hooker, W.J. 1864. Curtis's Bot. Mag. 20: plate 5469.

Jeffrey, C. 1962 ('1961'). Notes on Cucurbitaceae, including a proposed new classification of the family. Kew Bull. 15: 337-371. 
Jeffrey, C. 1979. Further notes on Cucurbitaceae IV. Some New-World taxa. Kew Bull. 33: 347394.

Jeffrey, C. 1980a. The Cucurbitaceae of Eastern Asia: 1-60. Roy. Bot. Gard., Kew.

Jeffrey, C. 1980b. Further notes on Cucurbitaceae V. The Cucurbitaceae of the Indian subcontinent. Kew Bull. 34: 789-809.

Jeffrey, C. 1982. Further notes on Cucurbitaceae VI. Cucurbitaceae of the Indian subcontinent: corrigenda and addenda. Kew Bull. 36: 737-740.

Jeffrey, C. \& W.J.J.O. de Wilde. 2006. A review of the subtribe Thladianthinae (Cucurbitaceae). Bot. Zhurn. (St. Petersburg) 91: 766-776.

Jeffrey, C. \& K. Kashyapa. 1960. The identity and nomenclature of Thladiantha dubia (Cucurbitaceae) of the 'Flora of British India'. Kew Bull. 14: 461-463.

Keraudren-Aymonin, M. 1975. Cucurbitacées. In: A. Aubréville \& J.-F. Leroy (eds.), Flore du Cambodge, du Laos et du Viêtnam 15: 1-114. Mus. Natl. Hist. Nat., Paris.

Léveillé, A.A.H. 1916. Catalogue des plantes du Yun-nan. Le Mans.

Li, J.Q. 1993. A revision of the genus Siraitia Merr. and two new genera of Cucurbitaceae. Acta Phytotax. Sin. 31: 45-55.

Li, J.Q. 1997. On the systematics of Thladiantha (Cucurbitaceae). Acta Bot. Yunnan. 19: 103-127.

Li, J.Q., C.Y. Wu \& A.M. Lu. 1992. New taxa of the genus Thladiantha (Cucurbitaceae). Acta Bot. Yunnan. 14: 133-134.

Liu, H.Y. 1993. Cucurbitaceae. In: Flora of Taiwan ed. 2, 3: 855-871. Taipei.

Lu, A.M. \& Zhi Y. Zhang. 1981. A revision of genus Thladiantha Bunge (Cucurbitaceae). Bull. Bot. Res., Harbin 1-2: 61-96.

Lu, A.M. \& Zhi Y. Zhang. 1984. The genus Siraitia Merr. in China. Guihaia 4: 27-33.

Lu, A.M. \& Zhi Y. Zhang. 1986a. Siraitia. In: A.M. Lu \& S.K. Chen (eds.), Fl. Reipubl. Popularis Sin. 73, 1: 161-167. Science Press, Beijing.

Lu, A.M. \& Zhi Y. Zhang. 1986b. Thladiantha. In: A.M. Lu \& S. K. Chen (eds.), Fl. Reipubl. Popularis Sin. 73, 1: 132-161. Science Press, Beijing.

Merrill, E.D. 1934. New Sumatran plants. I. Pap. Michigan Acad. Sci. 19: 199-201.

Merrill, E.D. 1937. Miscellanea Sinensia. Sunyatsenia 3: 261-262.

Merrill, E.D. 1940. Records of Indo-Chinese plants, II. J. Arnold Arbor. 21: 386.

Miquel, F. A.W. 1856. Flora Indiae Batavae 1, 1: 652-683. Van der Post, Amsterdam.

Pax, F.A. in E.G.O. Müller \& F. A. Pax. 1889. Cucurbitaceae. In: A. Engler \& K. Prantl, Die natürlichen Pflanzenfamilien 4, 5 (specieller Teil): 9-39. Engelmann, Leipzig.

Roemer, M.J. 1846. Peponiferarum. Syn. Monogr. 2: 1-118. Landes-Industrie-Comptoirs, Weimar.

Seringe, N.C. 1828. Cucurbitaceae. In: A.P. de Candolle, Prodromus systematis regni vegetabilis naturalis 3: 297-320. Treuttel \& Würtz, Paris.

Swingle, W.T. 1941. Momordica grosvenori sp. nov. the source of the Chinese Lo Han Kuo. J. Arnold Arbor. 22: 197-203.

Von Bunge, A.A. 1833. Cucurbitaceae. In: Enumeratio plantarum, quas in China boreali collegit.: 29. St. Petersburg.

Zhong, S.Q. \& D. Fang. 1984. Siraitia siamensis (Craib) C. Jeffrey - A new recorded species of Cucurbitaceae in Guangxi. Guihaia 4: 23-25.

\section{IDENTIFICATION LIST}

$$
\begin{aligned}
1 & =\text { Sinobaijiania smitinandii } \\
2 & =\text { Siraitia grosvenorii } \\
3 \mathrm{a} & =\text { Siraitia siamensis } \text { var. siamensis } \\
3 \mathrm{~b} & =\text { Siraitia siamensis } \text { var. silomaradjae }
\end{aligned}
$$

$$
\begin{aligned}
& 4=\text { Thladiantha angustisepala } \\
& 5=\text { Thladiantha cordifolia } \\
& 6=\text { Thladiantha hookeri } \\
& 7=\text { Thladiantha indochinensis } \\
& 8=\text { Thladiantha tonkinensis }
\end{aligned}
$$

Backer 5937: 3a - Bakhuizen van den Brink Sr. 1744: 5; 1748: 5; 4773: 5 - Balansa 2002: 3a; 4003: 8; 4004: 8; 4549: 8 - Bartlett 8702a: 3b - Beumée 1928: 5 - Blume 1464: 5 - Bunchuai 1702: 6 - Bünnemeijer 520: 5; 529: 5; 529a: 5; 3558: 5; 3740: 5; 4303: 5 . 
Cuong 1083: 6; NMC 1774: 3a; NMC 1783: 3a.

De Wilde 21800A: 5; 22127: 1; 22132: 1; 22170: 6; 22298: 1; 22306: 2; SAN 139471: 3a; SAN 144097: 3a; SAN 144528: 3a; SAN 144788: 3a; SAN 151203: 3a - Docters van Leeuwen s.n., 28-09-1910: 5; 231: 5 - Dorgelo 8558: 5.

Eberhardt 4273: 3a.

Gadoh anak Umbai KL 764: 3a; KL 1468: 3a; KL 1600: 3a - Garrett 692: 6; 1096: 6; 1414: 6 - Geesink 5923: 5; 6161: 6; 6733: 3a.

Hochreutiner 1255: 5 - Hooker \& Thomson 1703: 6; 2057: 5 - Huq 4314: 5.

Junghuhn 875: 5 .

Katakura 2004 27: 5; 2004 29: 5 - Kerr 1171: 3a; 2449: 4; 6338: 6 - Koonthunthod 538: 1 - Koorders 23125: 5; 23925: 5; 26285: 5; 29200: 5; 43258: 5 .

Larsen 32487: 3a; 43585: 6; 46169: 4 - Liang Xiangri 67217: 5 - Lörzing 5089: 5; 5595: 3a; 13093: 5 - Luo lin-bo 131: 5 .

Maxwell 85-1028: 3a; 88-996: 6; 88-1236: 6; 89-799: 6; 89-1356: 6; 90-860: 6; 91-679: 6; 93-765: 6; 93-890: 5; 96-883: 6; 96-912: 6; 99-96: 5; 01-523: 6; 05-138: 4 - Middleton 147: 6 - Murata T 16913: 6.

Palee 67: 6 - Panatkool 155: 6 - Panyarat 131: 1; s.n., 21-07-2004: 1 - Parnell 95-189: 6; 95-516: 4 - Pételot 2185: 7; 2194: 7; 8414: 3a - Petrmitr 13: 6 - Phonsena 3747: 1; 3802: 1; 3819: 1; 3820: 1; 3903: 1; 3910: 1; 3932: 3a; 3949B: 3a; 3960: 4; 3961: 4; 3964: 4; 4225: 3a; 4563: 3a; 4588: 1; 4608: 3a; 4610: 1; 4629: 1; 4636: 4; 4637: 4; 4680: 4; 4681: 4; 5204: 4; 5238: 4; s.n. 17-06-2003: 1; s.n. 29-09-2006: 1 - Poilane 7730: 3a; 20483: 8; 26355: 4 - Pooma 361: 6; 1253: 4; 2689: 1 ; 2825: 6; 5707: 5 - Put 18: $1 ; 127: 5$.

Raap 177: 5 - Rao 7553: 5; 10621: 5.

Sai Jai 21: 6 - Santisuk 103: 8; 269: 5; 6923: 8 - Smitinand 8136: 1; 8874: 6 - Sørensson 3934: 6 - Srisanga QBG 21179: 8.

Takahashi T 62855: 6 - Tsi Zhanhuo 91-461: 6; 92-118: 8.

Van Beusekom 918: 3a; 950: 6; 1311: 6; 4652: 4 - Van Steenis 3961: 5 - Vidal 5175: 7.

Wallich Cat. 6740A: 5 - Watt 6740: 5 - Wichian 197: 5 - Wieringa 3417: 5 - Winit 1739: 6 - Wisse 1218: 5 - Wongprasert 997-34: 4; 45-97: 6; SN 120885: 5.

Zhang Guicai 445: 2.

\section{INDEX}

Accepted names are in roman type, new species and combination in bold and synonyms in italics. The numbers behind the species names are the combination of genus number - species number.

Baijiania auct. [p. 494]

Gymnopetalum horsfieldii Miq. 3-2 piperifolium Miq. 3-2

Hemsleya tonkinensis Cogn. 3-3 trifoliolata Cogn. 3-3 yunnanensis Cogn. 3-3

Luffa cordifolia Blume 3-2

Momordica grosvenorii Swingle 2-1 tonkinensis Gagnep. 2-2

Neoluffa Chakrav. [p. 499]

Sinobaijiania C. Jeffrey \& W.J. de Wilde [p. 494]

smitinandii W.J. de Wilde \& Duyfjes 1-1

Siraitia Merr. [p. 499] subg. Microlagenaria auct. [p. 494] grosvenorii (Swingle) A.M. Lu \& Zhi Y. Zhang 2-1
(Siraitia) siamensis (Craib) S.Q. Zhong \& D. Fang 2-2 var. siamensis $2-2 \mathrm{a}$

var. silomaradjae (Merr.) W.J. de Wilde \& Duyfjes 2-2b

Siraitia auct. [p. 494]

Thladiantha Bunge [p. 503]

subg. Microlagenaria C. Jeffrey [p. 499]

angustisepala W.J. de Wilde \& Duyfjes 3-1

calcarata Cogn. 3-2

var. subglabra Cogn. 3-2

var. tonkinensis Cogn. 3-5

calcarata auct. 3-5

cordifolia (Blume) Cogn. 3-2

var. tomentosa A.M. Lu \& Zhi Y. Zhang 3-1

forma glabrescens Hochr. 3-2 
(Thladiantha)

cordifolia auct. 3-5

digitata H. Lév. 3-3

dubia auct. 3-2

grosvenorii (Swingle) C. Jeffrey 2-1

heptadactyla Cogn. 3-3

hookeri C.B. Clarke 3-3

var. heptadactyla (Cogn.) A.M. Lu \& Zhi Y. Zhang 3-3

var. pentadactyla (Cogn.) A.M. Lu \& Zhi Y. Zhang 3-3
(Thladiantha)

indochinensis Merr. 3-4

pentadactyla Cogn. 3-3

siamensis Craib 2-2

tonkinensis Gagnep. 3-5

trifoliolata (Cogn.) Merr. 3-3

Trichosanthes javanica Miq. 3-2 